TRANSACTIONS OF THE

AMERICAN MATHEMATICAL SOCIETY

Volume 352, Number 12, Pages 5405-5433

S 0002-9947(00)02486-7

Article electronically published on June 28, 2000

\title{
COMPACT NILMANIFOLDS WITH NILPOTENT COMPLEX STRUCTURES: DOLBEAULT COHOMOLOGY
}

\author{
LUIS A. CORDERO, MARISA FERNÁNDEZ, ALFRED GRAY, AND LUIS UGARTE
}

\begin{abstract}
We consider a special class of compact complex nilmanifolds, which we call compact nilmanifolds with nilpotent complex structure. It is shown that if $\Gamma \backslash G$ is a compact nilmanifold with nilpotent complex structure, then the Dolbeault cohomology $H_{\bar{\partial}}^{*, *}(\Gamma \backslash G)$ is canonically isomorphic to the $\bar{\partial}$-cohomology $H_{\bar{\partial}}^{*, *}\left(\mathfrak{g}^{\mathbb{C}}\right)$ of the bigraded complex $\left(\Lambda^{*, *}\left(\mathfrak{g}^{\mathbb{C}}\right)^{*}, \bar{\partial}\right)$ of complex valued left invariant differential forms on the nilpotent Lie group $G$.
\end{abstract}

\section{INTRODUCTION}

During the last few years compact complex nilmanifolds have proved to be very useful in producing a rich and wide variety of examples of compact complex manifolds possessing "unusual" properties. For example, it has been proved that any compact complex nilmanifold, not a torus, carries no positive definite Kähler metric [BG], [CFG2, [Ha; nevertheless, many of these manifolds carry symplectic forms, or indefinite Kähler metrics with remarkable curvature properties AFGM]. Also, examples of compact complex manifolds for which the Frölicher spectral sequence $[\mathrm{F}$ associated to their complex structure does not collapse at the second level have been constructed using compact complex nilmanifolds [CFG3], [CFG4, CFGU.

By compact complex nilmanifold we mean a complex manifold of the form $\Gamma \backslash G$, where $G$ is a (real) simply-connected connected nilpotent Lie group possessing a left invariant integrable almost complex structure, and $\Gamma$ is a lattice of $G$ of maximal rank; thus $\Gamma \backslash G$ inherits its complex structure from that of $G$ by passing to the quotient. For a compact complex nilmanifold $\Gamma \backslash G$, of (complex) dimension $n$, there is a (complex) basis $\left\{\omega_{i} ; 1 \leq i \leq n\right\}$ of forms of type $(1,0)$, such that the equations

$$
d \omega_{i}=\sum_{j<k \leq n} A_{i j k} \omega_{j} \wedge \omega_{k}+\sum_{j, k \leq n} B_{i j k} \omega_{j} \wedge \bar{\omega}_{k} \quad(1 \leq i \leq n)
$$

\footnotetext{
Received by the editors March 29, 1998 and, in revised form, December 20, 1998.

2000 Mathematics Subject Classification. Primary 32L05, 55P62, 57T15; Secondary 53C56, $32 \mathrm{Q} 99$.

Partially supported by Xunta de Galicia (Spain), Research Project XUGA20701B93, U.P.V. Project 127.310-EA 191/94, DGICYT Projects PB94-0633-C02-01 and PB94-0633-C02-02, and by DGICYT Sabbatical Program 1995-0735.

The revised version was submitted just after the unexpected death of A. Gray in Bilbao (Spain) on October 27, 1998.
} 
hold. Here $A_{i j k}$ and $B_{i j k}$ are constants. Equations (1) can be very complicated. But there are many examples of compact complex nilmanifolds for which the equations (1) reduce to

$$
d \omega_{i}=\sum_{j<k<i} A_{i j k} \omega_{j} \wedge \omega_{k}+\sum_{j, k<i} B_{i j k} \omega_{j} \wedge \bar{\omega}_{k} \quad(1 \leq i \leq n) .
$$

In this paper we consider those compact complex nilmanifolds $\Gamma \backslash G$ that can be defined by equations (2), and we call such a compact complex nilmanifold a compact nilmanifold with nilpotent complex structure. If $G$ is indeed a complex Lie group, then $\Gamma \backslash G$ is a compact complex parallelizable nilmanifold in the sense of H.C. Wang Wa]. The compact complex parallelizable nilmanifolds are precisely those compact nilmanifolds with nilpotent complex structure for which the coefficients $B_{i j k}$ in (2) vanish.

It is well known that for any Lie group there is the ascending central series $\left\{\mathfrak{g}_{l} ; l \geq 0\right\}$, associated to the Lie algebra $\mathfrak{g}$ of $G$, which characterizes the nilpotent Lie groups. In order to distinguish the compact nilmanifolds $\Gamma \backslash G$ with nilpotent complex structure, we define a new series $\left\{\mathfrak{a}_{l} ; l \geq 0\right\}$ associated to a nilpotent Lie group $G$ with a left invariant integrable almost complex structure that is useful in finding a condition equivalent to that given by the equations (2) (see Section 2, Theorem 12 and Theorem 13).

One of the main tools in the proofs of the results we have mentioned is a well known theorem, due to Nomizu [No], which asserts that the cohomology $H^{*}(\mathfrak{g})$ of the complex $\Lambda^{*} \mathfrak{g}^{*}$ of left invariant differential forms on the nilpotent Lie group $G(\mathfrak{g}$ $=$ Lie algebra of $G$ ) is isomorphic to the de Rham cohomology $H^{*}(\Gamma \backslash G, \mathbb{R}$ ). (Nomizu established an additive isomorphism, but there actually exists a multiplicative isomorphism.) However, no similar result seems to be known in the general case for the Dolbeault cohomology $H_{\bar{\partial}}^{*, *}(\Gamma \backslash G)$ either of a compact complex nilmanifold or of a compact nilmanifold with nilpotent complex structure. Sakane $\mathrm{Sa}$ proved a theorem for the Dolbeault cohomology of a compact complex parallelizable nilmanifold $\Gamma \backslash G$; but there exist many interesting compact nilmanifolds with a nilpotent complex structure which are not complex parallelizable but only real parallelizable (see Examples 2-4 in Section 5). Our purpose is to prove the following theorem.

Main Theorem. Let $\Gamma \backslash G$ be a compact nilmanifold with a nilpotent complex structure, and let $\mathfrak{g}$ be the Lie algebra of $G$. Then there is a canonical isomorphism

$$
H_{\bar{\partial}}^{p, q}(\Gamma \backslash G) \cong H_{\bar{\partial}}^{p, q}\left(\mathfrak{g}^{\mathbb{C}}\right),
$$

where $H_{\bar{\partial}}^{*, *}\left(\mathfrak{g}^{\mathbb{C}}\right)$ denotes the cohomology ring of the differential bigraded algebra $\Lambda^{*, *}\left(\mathfrak{g}^{\mathbb{C}}\right)^{*}$, associated to the complexified Lie algebra $\mathfrak{g}^{\mathbb{C}}$, with respect to the operator $\bar{\partial}$ in the canonical decomposition $d=\partial+\bar{\partial}$ of the Chevalley-Eilenberg differential in $\Lambda^{*}\left(\mathfrak{g}^{\mathbb{C}}\right)^{*}$.

Sakane's theorem follows as a corollary.

An interesting question remains open: does the Main Theorem hold for an arbitrary compact complex nilmanifold?

The paper is structured as follows. In Section 2, we find necessary conditions for a compact nilmanifold to have a nilpotent complex structure (see Proposition 10). Then in Section 3 we describe how a compact nilmanifold $\Gamma \backslash G$ with a nilpotent 
complex structure can be realized as the total space at the top of a tower of holomorphic principal bundles with complex tori as structure groups, each base manifold in the tower being a compact nilmanifold with a nilpotent complex structure. Moreover, it is shown that each bundle in this tower carries a canonical principal connection. In Section 4, we use Borel's spectral sequence [Hi] as the main tool for the proof of a Hirsch Lemma which allows us to determine a minimal model (in the sense of [NT]) for the Dolbeault cohomology of the total space of a holomorphic fibre bundle satisfying some suitable hypothesis. This Hirsch Lemma, together with the construction of the tower in the preceding section, leads to a proof of our Main Theorem. Section 5 is devoted to the discussion of examples illustrating the constructions and results of the previous sections. Finally, in Section 6 we give examples of compact complex nilmanifolds $L^{6}$ and $M^{10}$ with complex structures not nilpotent (the example $L^{6}$ is due to E. Abbena, S. Garbiero and S. Salamon). Also, in Section 6, by using the results of Section 2, we prove that the compact nilmanifold $L^{6}$ admits no nilpotent complex structures.

Acknowledgment. The authors are grateful to Simon Salamon for several very useful discussions, and in particular for calling our attention to the existence of the compact complex nilmanifold $L^{6}$.

\section{Compact Nilmanifolds With nilpotent COMPleX STRUCTURE}

Let us start by distinguishing those compact complex nilmanifolds that admit a nilpotent complex structure.

Let $G$ be a real nilpotent Lie group. Instead of describing the Lie algebra $\mathfrak{g}$ of $G$ in terms of its bracket, we shall use the exterior differential on the dual space $\mathfrak{g}^{*}$. The two are equivalent because $d \alpha(X, Y)=-\alpha([X, Y])$, where $\alpha \in\left(\mathfrak{g}^{\mathbb{C}}\right)^{*}$ and $X, Y \in \mathfrak{g}^{\mathbb{C}}$.

Let us suppose that $G$ has a left invariant almost complex structure, and choose a complex basis $\left\{\omega_{1}, \ldots, \omega_{n}\right\}, 2 n=\operatorname{dim} G$, for the complex forms on $\mathfrak{g}^{\mathbb{C}}$. Then $\left\{\omega_{1}, \bar{\omega}_{1}, \ldots, \omega_{n}, \bar{\omega}_{n}\right\}$ is a real basis for $\mathfrak{g}^{*}$. Thus the structure equations sufficient to determine the bracket in $\mathfrak{g}$ are

$$
d \omega_{i}=\sum_{j<k} A_{i j k} \omega_{j} \wedge \omega_{k}+\sum_{j, k} B_{i j k} \omega_{j} \wedge \bar{\omega}_{k}+\sum_{j<k} C_{i j k} \bar{\omega}_{j} \wedge \bar{\omega}_{k} .
$$

If we want $\mathfrak{g}$ to be the Lie algebra of a nilpotent Lie group with a left invariant integrable almost complex structure, it is necessary and sufficient that the coefficients $C_{i j k}$ in (3) vanish; that is, the structure equations for $\mathfrak{g}$ are of the form (1). (See for example [KN vol. II, Theorem 2.8].)

Let $G$ be a simply-connected connected $s$-step nilpotent Lie group with Lie algebra $\mathfrak{g}$. The ascending central series of $\mathfrak{g}$ is defined as follows. Let $\mathfrak{g}_{l}, l \geq 0$, be defined inductively by

$$
\mathfrak{g}_{0}=0, \quad \mathfrak{g}_{l}=\left\{X \in \mathfrak{g} \mid[X, \mathfrak{g}] \subseteq \mathfrak{g}_{l-1}\right\}, \quad l \geq 1 ;
$$

then each $\mathfrak{g}_{l}$ is an ideal in $\mathfrak{g}, \mathfrak{g}_{1}$ is the center of $\mathfrak{g}, \mathfrak{g}_{1} \neq 0, \mathfrak{g}_{s}=\mathfrak{g}, \mathfrak{g}_{l} \subseteq \mathfrak{g}_{l+1}$, and $\operatorname{dim} \mathfrak{g}_{l}<\operatorname{dim} \mathfrak{g}_{l+1}$ for $0 \leq l \leq s-1$. The increasing sequence

$$
\mathfrak{g}_{0}=0 \hookrightarrow \mathfrak{g}_{1} \hookrightarrow \mathfrak{g}_{2} \hookrightarrow \cdots \hookrightarrow \mathfrak{g}_{s-1} \hookrightarrow \mathfrak{g}_{s}=\mathfrak{g}
$$

is called the ascending central series of the Lie algebra $\mathfrak{g}$.

Let us now suppose that $G$ carries a left invariant integrable almost complex structure $J$. Then $\mathfrak{g}$ is a complex vector space, but in general not a complex Lie 
algebra. Moreover, each term $\mathfrak{g}_{l}$ is an ideal of $\mathfrak{g}$, so $\mathfrak{g}_{l}$ is a real subspace of $\mathfrak{g}$, but not necessarily a complex subspace of $\mathfrak{g}$. (In fact, in Section 5 , we shall give examples of (real) Lie algebras, each of which has the structure of complex vector space, for which the terms $\mathfrak{g}_{l}$ of the ascending central series do not admit a structure of complex vector subspace of $\mathfrak{g}$; see Examples 3 and 4.)

In order to distinguish and study a compact nilmanifold with a nilpotent complex structure, we introduce the following ascending series $\left\{\mathfrak{a}_{l}\right\}$ associated to the Lie algebra $\mathfrak{g}$.

Definition 1. The ascending series $\left\{\mathfrak{a}_{l} ; l \geq 0\right\}$ (compatible with the integrable almost complex structure $J$ of $G$ ) of $\mathfrak{g}$ is defined inductively by

$$
\mathfrak{a}_{0}=0, \quad \mathfrak{a}_{l}=\left\{X \in \mathfrak{g} \mid[X, \mathfrak{g}] \subseteq \mathfrak{a}_{l-1} \quad \text { and }[J X, \mathfrak{g}] \subseteq \mathfrak{a}_{l-1}\right\}, \quad l \geq 1
$$

It is easy to verify that $\mathfrak{a}_{l}$ is an ideal of $\mathfrak{g}$, a complex subspace of $\mathfrak{g}$ and $\mathfrak{a}_{l} \subseteq \mathfrak{a}_{l+1}$, for each $l \geq 0$; moreover, $\mathfrak{a}_{l} \subseteq \mathfrak{g}_{l}$ for each $l \geq 0$.

Lemma 2. If $\mathfrak{a}_{l}=\mathfrak{a}_{l+1}$ for some $l \geq 0$, then $\mathfrak{a}_{r}=\mathfrak{a}_{l}$ for all $r \geq l$.

Proof. Since $\mathfrak{a}_{l}=\mathfrak{a}_{l+1}$, it follows from (4) that

$$
\begin{aligned}
\mathfrak{a}_{l+2} & =\left\{X \in \mathfrak{g} \mid[X, \mathfrak{g}] \subseteq \mathfrak{a}_{l+1} \text { and }[J X, \mathfrak{g}] \subseteq \mathfrak{a}_{l+1}\right\} \\
& =\left\{X \in \mathfrak{g} \mid[X, \mathfrak{g}] \subseteq \mathfrak{a}_{l} \text { and }[J X, \mathfrak{g}] \subseteq \mathfrak{a}_{l}\right\} \\
& =\mathfrak{a}_{l+1}
\end{aligned}
$$

Thus, $\mathfrak{a}_{r}=\mathfrak{a}_{l+1}=\mathfrak{a}_{l}$ for all $r \geq l$.

From Lemma 2 we guess the possible existence of a series $\left\{\mathfrak{a}_{l} ; l \geq 0\right\}$ for which $\mathfrak{g} \neq \mathfrak{a}_{l}=\mathfrak{a}_{l+1}$ for some $l \geq 0$; in fact, in Section 6 , we give examples for which $\mathfrak{a}_{l}=0$ for all $l \geq 0$. In the present section we find a condition equivalent to having $\mathfrak{a}_{t}=\mathfrak{g}$ for some $t \geq 1$ and $\operatorname{dim} \mathfrak{a}_{l}<\operatorname{dim} \mathfrak{a}_{l+1}$ for $0 \leq l \leq t-1$ (see Theorem 12).

First, we state some general properties of the series $\left\{\mathfrak{a}_{l} ; l \geq 0\right\}$ in relation with the series $\left\{\mathfrak{g}_{l} ; l \geq 0\right\}$.

Lemma 3. Let $\mathfrak{g}$ be the Lie algebra of a simply-connected connected s-step nilpotent Lie group with a left invariant integrable almost complex structure $J$. Let $\left\{\mathfrak{a}_{l} ; l \geq 0\right\}$ be the ascending series defined by (4).

(i) If there is $l \geq 0$ such that $\mathfrak{a}_{l}=\mathfrak{g}_{l}$, then $J\left(\mathfrak{g}_{l}\right)=\mathfrak{g}_{l}$.

(ii) If $\mathfrak{a}_{l-1}=\mathfrak{g}_{l-1}$, for some $l>0$, then $\mathfrak{a}_{l}=\mathfrak{g}_{l}$ if and only if $J\left(\mathfrak{g}_{l}\right)=\mathfrak{g}_{l}$. $J$.

(iii) If $\mathfrak{a}_{l-1}=\mathfrak{g}_{l-1}$ then $\mathfrak{a}_{l}$ is the largest subspace of $\mathfrak{g}_{l}$ which is invariant under

Proof. Since $J\left(\mathfrak{a}_{l}\right)=\mathfrak{a}_{l}$ for $l \geq 0$, we obtain (i) and (ii) directly from the definitions of the series $\left\{\mathfrak{a}_{l} ; l \geq 0\right\}$ and $\left\{\mathfrak{g}_{l} ; l \geq 0\right\}$. To prove (iii), let us consider a subspace $V$ of $\mathfrak{g}_{l}$ invariant under $J$ and satisfying $\mathfrak{a}_{l} \subseteq V \subseteq \mathfrak{g}_{l}$. Since $J(V)=V \subseteq \mathfrak{g}_{l}$, we obtain that $[v, \mathfrak{g}] \subseteq \mathfrak{g}_{l-1}$ and $[J(v), \mathfrak{g}] \subseteq \mathfrak{g}_{l-1}$ for $v \in V$. But $\mathfrak{a}_{l-1}=\mathfrak{g}_{l-1}$ implies that $v \in \mathfrak{a}_{l}$; therefore, $\mathfrak{a}_{l}=V$.

Remark 4. We note that, from Lemma 3, if $J\left(\mathfrak{g}_{l}\right) \not \subset \mathfrak{g}_{l}$, for some $l>0$, then the inclusion $\mathfrak{a}_{l} \subset \mathfrak{g}_{l}$ is strict. Moreover, Lemma 3 also implies that if $\mathfrak{a}_{s-1}=\mathfrak{g}_{s-1}$, then $\mathfrak{a}_{s}=\mathfrak{g}_{s}=\mathfrak{g}$.

Since $\mathfrak{a}_{0}=\mathfrak{g}_{0}=0$, we obtain the following corollary from (ii) and (iii) of Lemma 3: 
Corollary 5. Under the conditions of Lemma 3 we have:

(i) The term $\mathfrak{a}_{1}$ is the largest subspace of the center $\mathfrak{g}_{1}$ of $\mathfrak{g}$ which is invariant under $J$.

(ii) If all the terms $\mathfrak{g}_{l}$ in the ascending central series $\left\{\mathfrak{g}_{l} ; l \geq 0\right\}$ of $\mathfrak{g}$ are invariant under $J$, then $\mathfrak{a}_{l}=\mathfrak{g}_{l}$ for each $l \geq 0$. In particular, $\mathfrak{a}_{s}=\mathfrak{g}_{s}=\mathfrak{g}$.

Suppose that $\Gamma \backslash G$ is a compact complex parallelizable nilmanifold in the sense of Wang Wa]; then $\mathfrak{g}$ is a complex Lie algebra, each $\mathfrak{g}_{l}$ is a complex Lie subalgebra of $\mathfrak{g}$, and from Lemma 3 we get $J\left(\mathfrak{g}_{l}\right)=\mathfrak{g}_{l}$ and $\mathfrak{a}_{l}=\mathfrak{g}_{l}$ for all $l \geq 0$. Therefore, the series $\left\{\mathfrak{a}_{l}\right\}$ and $\left\{\mathfrak{g}_{l}\right\}$ are the same for compact complex parallelizable nilmanifolds.

There are examples of nilpotent (non-complex) Lie algebras $\mathfrak{g}$ for which $\mathfrak{a}_{l}=\mathfrak{g}_{l}$ for each $l \geq 0$ (see Section 5, Example 2). However, there are also examples of nilpotent Lie algebras $\mathfrak{g}$ for which $\operatorname{dim} \mathfrak{a}_{l}<\operatorname{dim} \mathfrak{g}_{l}$ for some $l>0$ (see Section 5, Examples 3 and 4).

Let us recall that the descending central series $\left\{\mathfrak{g}^{k} ; k \geq 0\right\}$ of $\mathfrak{g}$ is defined inductively by

$$
\mathfrak{g}^{0}=\mathfrak{g}, \quad \mathfrak{g}^{k}=\left[\mathfrak{g}^{k-1}, \mathfrak{g}\right], \quad k \geq 1 .
$$

It is known $\mathrm{Va}$, page 191] that $\mathfrak{g}$ is an $s$-step nilpotent Lie algebra if and only if $\mathfrak{g}^{s}=0$ and $\mathfrak{g}^{s-1} \neq 0$. Moreover, in this case the sequence $\left\{\mathfrak{g}^{k} ; k \geq 0\right\}$ is a sequence of ideals of $\mathfrak{g}$ such that $\operatorname{dim} \mathfrak{g}^{k}>\operatorname{dim} \mathfrak{g}^{k+1}$, for $0 \leq k \leq s-1$. We next state some general properties for the series $\left\{\mathfrak{a}_{l} ; l \geq 0\right\}$ with respect to the series $\left\{\mathfrak{g}^{k} ; k \geq 0\right\}$.

Lemma 6. Under the conditions of Lemma 3 , let $\left\{\mathfrak{a}_{l} ; l \geq 0\right\}$ be the ascending series associated to $\mathfrak{g}$ defined by (4). Then:

(i) If $\mathfrak{g}^{k} \subset \mathfrak{a}_{l}$ and $J\left(\mathfrak{g}^{k-1}\right)=\mathfrak{g}^{k-1}$ for some $k \geq 0$ and some $l \geq 0$, then $\mathfrak{g}^{k-1} \subset \mathfrak{a}_{l+1}$.

(ii) If $[\mathfrak{g}, \mathfrak{g}] \subset \mathfrak{a}_{l}$ for some $l \geq 0$, then $\mathfrak{a}_{l+1}=\mathfrak{g}$.

Proof. Let $X \in \mathfrak{g}^{k-1}$. Then $[X, \mathfrak{g}] \subset\left[\mathfrak{g}^{k-1}, \mathfrak{g}\right]=\mathfrak{g}^{k}$. Moreover, since $J\left(\mathfrak{g}^{k-1}\right)=$ $\mathfrak{g}^{k-1}, J X \in \mathfrak{g}^{k-1}$. Therefore, from (5) it follows that $[J X, \mathfrak{g}] \subset\left[\mathfrak{g}^{k-1}, \mathfrak{g}\right]=\mathfrak{g}^{k}$. Now, since $\mathfrak{g}^{k} \subset \mathfrak{a}_{l}$ by hypothesis, we obtain the following inclusions: $[X, \mathfrak{g}] \subset \mathfrak{g}^{k} \subset \mathfrak{a}_{l}$ and $[J X, \mathfrak{g}] \subset \mathfrak{g}^{k} \subset \mathfrak{a}_{l}$. This implies, using (4), that $X \in \mathfrak{a}_{l+1}$. Therefore, $X \in \mathfrak{a}_{l+1}$ for each $X \in \mathfrak{g}^{k-1}$, which proves (i).

To prove (ii), we notice that $[\mathfrak{g}, \mathfrak{g}]=\mathfrak{g}^{1}$ and $J\left(\mathfrak{g}^{0}\right)=\mathfrak{g}^{0}$, because $\mathfrak{g}^{0}=\mathfrak{g}$. Then, using (i) for $k=1$, we obtain that $[\mathfrak{g}, \mathfrak{g}] \subset \mathfrak{a}_{l}$ implies $\mathfrak{g}=\mathfrak{g}^{0} \subset \mathfrak{a}_{l+1}$.

Corollary 7. If all the terms $\mathfrak{g}^{k}$ in the descending central series $\left\{\mathfrak{g}^{k} ; k \geq 0\right\}$ of $\mathfrak{g}$ are invariant under $J$, then $\mathfrak{a}_{s}=\mathfrak{g}_{s}=\mathfrak{g}$.

Proof. Since $\mathfrak{g}$ is $s$-step nilpotent, $\mathfrak{g}^{s}=\mathfrak{a}_{0}=0$. Using Lemma 6 (i) for $k=s$ and $l=0$, we obtain $\mathfrak{g}^{s-1} \subset \mathfrak{a}_{1}$ because $J\left(\mathfrak{g}^{s-1}\right)=\mathfrak{g}^{s-1}$. Since $J\left(\mathfrak{g}^{s-2}\right)=\mathfrak{g}^{s-2}$, using again Lemma 6 (i) with $k=s-1$ and $l=1$, we obtain $\mathfrak{g}^{s-2} \subset \mathfrak{a}_{2}$, and continuing in this way we arrive at $\mathfrak{g}^{2} \subset \mathfrak{a}_{s-2}$. Since $J\left(\mathfrak{g}^{1}\right)=\mathfrak{g}^{1}$, using Lemma 6 (i) with $k=2$ and $l=s-2$, we obtain $\mathfrak{g}^{1}=[\mathfrak{g}, \mathfrak{g}] \subset \mathfrak{a}_{s-1}$. Finally, from Lemma 6 (ii) it follows that $\mathfrak{a}_{s}=\mathfrak{g}$.

Definition 8. Let $G$ be a simply-connected connected $s$-step nilpotent Lie group with Lie algebra $\mathfrak{g}$. Let us suppose that $J$ is a left invariant integrable almost complex structure on $G$.

(i) We shall say that $J$ is a nilpotent (left invariant) complex structure if $\mathfrak{a}_{t}=\mathfrak{g}$ for some $t>0$. 
(ii) Furthermore, if $J$ is a nilpotent (left invariant) complex structure on $G$, and $\Gamma$ is a lattice of $G$ of maximal rank, we shall say that the compact nilmanifold $\Gamma \backslash G$ (with the complex structure defined from $J$ by passing to the quotient) has a nilpotent complex structure.

In order to formulate the existence of a nilpotent complex structure on the compact nilmanifold $\Gamma \backslash G$ in terms of the structure equations of $G$, we need the following:

Lemma 9. Let $G$ be a simply-connected connected s-step nilpotent Lie group with Lie algebra $\mathfrak{g}$. Suppose that $G$ carries a left invariant integrable almost complex structure $J$, for which there is a (complex) basis $\left\{\omega_{i} ; 1 \leq i \leq n\right\}$ of forms of type $(1,0)$ that satisfy equations (2). Let $\left\{Z_{i}, \bar{Z}_{i} ; 1 \leq i \leq n\right\}$ be the basis of $\mathfrak{g}$ dual to the basis $\left\{\omega_{i}, \bar{\omega}_{i} ; 1 \leq i \leq n\right\}$ of $\mathfrak{g}^{*}$. For each $1 \leq i \leq n$, let us denote $X_{i}=\mathfrak{R e}\left(Z_{i}\right)$ and $Y_{i}=\mathfrak{I m}\left(Z_{i}\right)$. Then, for $1 \leq l \leq n$, the term $\mathfrak{a}_{l}$ in the series $\left\{\mathfrak{a}_{l} ; l \geq 0\right\}$ has at least the following generators: $X_{n-l+1}, Y_{n-l+1}, \ldots, X_{n}, Y_{n}$.

Proof. Due to the fact that each $d \omega_{i}$ is a linear combination of wedge products of $\omega_{j}$ 's and their conjugates with $j<i$, it follows in particular that $\left[U, Z_{n}\right]=\left[U, \bar{Z}_{n}\right]=$ 0 for every $U \in \mathfrak{g}$. Therefore, $Z_{n}, \bar{Z}_{n} \in \mathfrak{a}_{1}$. This implies that $X_{n}=\mathfrak{R e}\left(Z_{n}\right)$ and $Y_{n}=\mathfrak{I m}\left(Z_{n}\right)=-J X_{n}$ both belong to $\mathfrak{a}_{1}$, which proves the assertion for $l=1$.

Suppose now that $X_{n-l+1}, Y_{n-l+1}, \ldots, X_{n}, Y_{n}$ are generators of $\mathfrak{a}_{l}$ with $1<l<$ $n$; then we shall show that $X_{n-l}, Y_{n-l}, X_{n-l+1}, Y_{n-l+1}, \ldots, X_{n}, Y_{n}$ are generators of $\mathfrak{a}_{l+1}$. For that, it suffices to prove that $Z_{n-l}, \bar{Z}_{n-l} \in \mathfrak{a}_{l+1}$. But, from equations (2) we get

$$
\begin{aligned}
{\left[Z_{j}, Z_{n-l}\right] } & =-\sum_{i=n-l+1}^{n} A_{i j n-l} Z_{i}, \quad 1 \leq j \leq n-l-1, \\
{\left[Z_{j}, \bar{Z}_{n-l}\right] } & =\sum_{i=n-l+1}^{n}\left(-B_{i j n-l} Z_{i}+\bar{B}_{i n-l j} \bar{Z}_{i}\right), \quad 1 \leq j \leq n-l,
\end{aligned}
$$

and these identities imply that $Z_{n-l}, \bar{Z}_{n-l} \in \mathfrak{a}_{l+1}$, which completes the proof.

It must be remarked that, under the conditions of Lemma 9, there exists always an integer $t$ with $1 \leq t \leq n$ such that $\mathfrak{a}_{t}=\mathfrak{g}$; in fact, at least $\mathfrak{a}_{n}=\mathfrak{g}$ always. Hence, and as a consequence of Lemma 2 and Lemma 9, we obtain the possible values for $t$ such that $\operatorname{dim} \mathfrak{a}_{t-1}<\operatorname{dim} \mathfrak{a}_{t}$ and $\mathfrak{a}_{t}=\mathfrak{g}$.

Proposition 10. In the conditions of Lemma 9, we have:

(i) Let $\mathfrak{a}_{l}$ be a term in the series $\left\{\mathfrak{a}_{l} ; l \geq 0\right\}$ such that $\mathfrak{a}_{l} \neq \mathfrak{g}$. Then

$$
\operatorname{dim} \mathfrak{a}_{l+1} \geq 2+\operatorname{dim} \mathfrak{a}_{l} .
$$

(ii) Let $\left\{\mathfrak{g}_{l} ; l \geq 0\right\}$ be the ascending central series of $\mathfrak{g}$. Then, for $0 \leq l \leq s$,

$$
\operatorname{dim} \mathfrak{g}_{l} \geq \operatorname{dim} \mathfrak{a}_{l} \geq 2 l .
$$

(iii) There exists a unique integer $t$ with $s \leq t \leq n$ such that $\operatorname{dim} \mathfrak{a}_{t-1}<\operatorname{dim} \mathfrak{a}_{t}$ and $\mathfrak{a}_{t}=\mathfrak{g}$.

Proof. Suppose $\mathfrak{a}_{l} \neq \mathfrak{g}$. Then, from Lemma 2, it follows that $\mathfrak{a}_{l} \neq \mathfrak{a}_{l+1} \subseteq \mathfrak{a}_{n}=\mathfrak{g}$. Now, (i) is a direct consequence of Lemma 9, which also implies that $\operatorname{dim} \mathfrak{a}_{l} \geq 2 l$, and, since $\mathfrak{a}_{l} \subseteq \mathfrak{g}_{l}$, we conclude (ii). In order to prove (iii) it suffices to show that $s \leq t$. But this inequality is a direct consequence of (ii) and the inclusions $\mathfrak{a}_{s} \subseteq \mathfrak{g}_{s}=\mathfrak{g}=\mathfrak{a}_{t}$ 
In Section 5 we describe examples of nilpotent Lie algebras illustrating the variety of possibilities inherent in Proposition 10 (iii). Example 2 satisfies $s=t=n=2$; Example 1 satisfies $s=t<n$; in Example 3 it is $s=2<3=t=n$, and in Example 4 it is $s=3<4=t<5=n$.

Note that in all these examples the sequence $\left\{\mathfrak{a}_{l}\right\}$ always stops at the term $\mathfrak{a}_{t}$ with $t=s$ or $s+1$; however, we have neither found a general proof of this fact, nor examples of $s$-step nilpotent Lie algebras for which the sequence $\left\{\mathfrak{a}_{l}\right\}$ stops at a term $\mathfrak{a}_{t}$ with $t \geq s+2$.

Proposition 11. Under the conditions of Lemma 9, we have

$$
\operatorname{dim}[\mathfrak{g}, \mathfrak{g}] \leq 2 n-3
$$

Proof. From equations (2) we deduce in particular that

$$
d \omega_{1}=0 \quad \text { and } \quad d \omega_{2}=B_{211} \omega_{1} \wedge \bar{\omega}_{1}
$$

Therefore, $Z_{1}, \bar{Z}_{1} \notin[\mathfrak{g}, \mathfrak{g}]$. Moreover, if $B_{211}=0$, then $Z_{2}, \bar{Z}_{2} \notin[\mathfrak{g}, \mathfrak{g}]$. Otherwise, if $B_{211} \neq 0$, then, using $(6)$, we get that

$$
\left[Z_{1}, \bar{Z}_{1}\right]=-B_{211} Z_{2}+\bar{B}_{211} \bar{Z}_{2}+\sum_{k=3}^{n}\left(-B_{k 11} Z_{k}+\bar{B}_{k 11} \bar{Z}_{k}\right)
$$

that is, $\mathfrak{I m}\left(B_{211} Z_{2}\right)$ can be in $[\mathfrak{g}, \mathfrak{g}]$ but $\mathfrak{R e}\left(B_{211} Z_{2}\right) \notin[\mathfrak{g}, \mathfrak{g}]$. In any case, $\operatorname{dim}[\mathfrak{g}, \mathfrak{g}] \leq$ $2 n-3$.

Next, with the following two theorems, we shall characterize which compact nilmanifolds admit a nilpotent complex structure.

Theorem 12. Let $G$ be a simply-connected connected s-step nilpotent Lie group, of dimension $2 n$, with Lie algebra $\mathfrak{g}$. Suppose that $G$ carries a left invariant integrable almost complex structure $J$, and let $\left\{\mathfrak{a}_{l} ; l \geq 0\right\}$ be the ascending series of $\mathfrak{g}$ compatible with $J$. Then, there is a (complex) basis $\left\{\omega_{i} ; 1 \leq i \leq n\right\}$ of left invariant forms of type $(1,0)$ such that equations (2) are the structure equations of $G$ if and only if $J$ is nilpotent (in the sense of Definition 8 (i)) on $G$.

Proof. Let us suppose that equations (2) are satisfied for some (complex) basis $\left\{\omega_{i} ; 1 \leq i \leq n\right\}$. From Proposition 10 we know that there exists a unique $t$ satisfying $\operatorname{dim} \mathfrak{a}_{t-1}<\operatorname{dim} \mathfrak{a}_{t}$ and $\mathfrak{a}_{t}=\mathfrak{g} ;$ moreover $\operatorname{dim} \mathfrak{a}_{l}<\operatorname{dim} \mathfrak{a}_{l+1}$ for $0 \leq l \leq$ $t-1$.

Conversely, let us suppose that $\mathfrak{a}_{l}=\mathfrak{g}$ for some $l>0$. From Lemma 2 we can take $t$ as the smallest integer such that $\mathfrak{a}_{t}=\mathfrak{g}, \operatorname{dim} \mathfrak{a}_{t-1}<\operatorname{dim} \mathfrak{a}_{t}$ and $\operatorname{dim} \mathfrak{a}_{l}<\operatorname{dim} \mathfrak{a}_{l+1}$ for $0 \leq l \leq t-1$. Thus, we have the series $\mathfrak{a}_{0}=\{0\} \subset \mathfrak{a}_{1} \subset \mathfrak{a}_{2} \subset \cdots \subset \mathfrak{a}_{t-1} \subset$ $\mathfrak{a}_{t}=\mathfrak{g}$. This series induces the following sequence of (quotient) Lie algebras and homomorphisms:

$$
\mathfrak{g} \longrightarrow \mathfrak{g} / \mathfrak{a}_{1} \longrightarrow \cdots \longrightarrow \mathfrak{g} / \mathfrak{a}_{l-1} \stackrel{\pi_{l}}{\longrightarrow} \mathfrak{g} / \mathfrak{a}_{l} \longrightarrow \cdots \longrightarrow \mathfrak{g} / \mathfrak{a}_{t-1} \longrightarrow 0
$$

where each $\pi_{l}$ is surjective and $\operatorname{ker} \pi_{l}=\mathfrak{a}_{l} / \mathfrak{a}_{l-1}$.

Let $\mathfrak{a}_{l}$ be a term in the series $\left\{\mathfrak{a}_{l} ; l \geq 0\right\}$ such that $\mathfrak{a}_{l} \neq \mathfrak{g}$. Since $\mathfrak{g}$ is a nilpotent Lie algebra and $\mathfrak{a}_{l}$ an ideal in $\mathfrak{g}$, the quotient Lie algebra $\mathfrak{g} / \mathfrak{a}_{l}$ is also nilpotent. Let $G_{l}$ be the simply-connected connected nilpotent Lie group defined by the Lie algebra $\mathfrak{g} / \mathfrak{a}_{l}$. Since $J\left(\mathfrak{a}_{l}\right)=\mathfrak{a}_{l}$, there exists on $G_{l}$ the left invariant almost complex structure $J_{l}$ induced by $J$ and given by

$$
J_{l}(\widetilde{X})=\widetilde{J X}
$$


for $\tilde{X} \in \mathfrak{g} / \mathfrak{a}_{l}$. (Here, we denote by $\widetilde{X}$ the element in $\mathfrak{g} / \mathfrak{a}_{l}$ defined by $\left.X \in \mathfrak{g}.\right)$ This structure $J_{l}$ is integrable on $G_{l}$ because the Nijenhuis tensor $N_{J_{l}}$ of $J_{l}$ satisfies $N_{J_{l}}(\widetilde{X}, \widetilde{Y})=\widetilde{N_{J}} \widetilde{X, Y)}$; thus, $N_{J_{l}}=0$.

Now, in order to construct a basis $\left\{\omega_{i} ; 1 \leq i \leq n\right\}$ satisfying equations (2), we start an iterative procedure from the Lie algebra $\mathfrak{g} / \mathfrak{a}_{t-1}$ of the sequence (7). Definition 1 implies that $\left[\mathfrak{g} / \mathfrak{a}_{t-1}, \mathfrak{g} / \mathfrak{a}_{t-1}\right]=0$, that is, $\mathfrak{g} / \mathfrak{a}_{t-1}$ is Abelian. Moreover, if we set $\operatorname{dim} \mathfrak{a}_{t-1}=2 n_{t-1}$, then $\operatorname{dim}\left(\mathfrak{g} / \mathfrak{a}_{t-1}\right)=2\left(n-n_{t-1}\right)$. Therefore, we can consider a basis $\left\{X_{1}, \bar{X}_{1}, \ldots, X_{n-n_{t-1}}, \bar{X}_{n-n_{t-1}}\right\}$ for $\mathfrak{g} / \mathfrak{a}_{t-1}$ such that

$$
\left[X_{i}, X_{j}\right]=\left[X_{i}, \bar{X}_{j}\right]=\left[\bar{X}_{i}, \bar{X}_{j}\right]=0
$$

in $\mathfrak{g} / \mathfrak{a}_{t-1}$ and for any $1 \leq i, j \leq n-n_{t-1}$. Let $\left\{\omega_{1}, \bar{\omega}_{1}, \ldots, \omega_{n-n_{t-1}}, \bar{\omega}_{n-n_{t-1}}\right\}$ be the real basis for $\left(\mathfrak{g} / \mathfrak{a}_{t-1}\right)^{*}$ dual to the basis $\left\{X_{1}, \bar{X}_{1}, \ldots, X_{n-n_{t-1}}, \bar{X}_{n-n_{t-1}}\right\}$. In terms of $\left\{\omega_{i}, \bar{\omega}_{i} ; 1 \leq i \leq n-n_{t-1}\right\}$, the identities (9) become

$$
d \omega_{i}=0, \quad 1 \leq i \leq n-n_{t-1} .
$$

Now, consider the Lie algebra $\mathfrak{g} / \mathfrak{a}_{t-2}$ in the sequence $(7)$. Then, we extend the basis $\left\{X_{1}, \bar{X}_{1}, \ldots, X_{n-n_{t-1}}, \bar{X}_{n-n_{t-1}}\right\}$ of $\mathfrak{g} / \mathfrak{a}_{t-1}$ to a basis $\left\{X_{1}, \bar{X}_{1}, \ldots, X_{n-n_{t-1}}\right.$, $\left.\bar{X}_{n-n_{t-1}}, \ldots, X_{n-n_{t-2}}, \bar{X}_{n-n_{t-2}}\right\}$ of $\mathfrak{g} / \mathfrak{a}_{t-2}$ in such way that $\left\{X_{n-n_{t-1}+1}, \bar{X}_{n-n_{t-1}+1}\right.$, $\left.\ldots, X_{n-n_{t-2}}, \bar{X}_{n-n_{t-2}}\right\}$ is a basis for $\mathfrak{a}_{t-1} / \mathfrak{a}_{t-2}$; in fact,

$$
\mathfrak{g} / \mathfrak{a}_{t-1} \cong\left(\mathfrak{g} / \mathfrak{a}_{t-2}\right) /\left(\mathfrak{a}_{t-1} / \mathfrak{a}_{t-2}\right)
$$

But $\mathfrak{a}_{t-1} / \mathfrak{a}_{t-2}$ is contained in the center of $\mathfrak{g} / \mathfrak{a}_{t-2}$, because $\left[\mathfrak{a}_{t-1} / \mathfrak{a}_{t-2}, \mathfrak{g} / \mathfrak{a}_{t-2}\right]=0$, and so we have

$$
\left[X_{i}, X_{k}\right]=\left[\bar{X}_{i}, X_{k}\right]=\left[X_{k}, X_{p}\right]=\left[\bar{X}_{k}, X_{p}\right]=\left[\bar{X}_{k}, \bar{X}_{p}\right]=0
$$

in $\mathfrak{g} / \mathfrak{a}_{t-2}$ for $1 \leq i \leq n-n_{t-1}$, and for $n-n_{t-1}+1 \leq k, p \leq n-n_{t-2}$; but in $\mathfrak{g} / \mathfrak{a}_{t-2}$ it happens that

$$
\left[X_{i}, X_{j}\right] \in \mathfrak{a}_{t-1} / \mathfrak{a}_{t-2} \text { for } 1 \leq i, j \leq n-n_{t-1} .
$$

Let $\left\{\omega_{1}, \bar{\omega}_{1}, \ldots, \omega_{n-n_{t-2}}, \bar{\omega}_{n-n_{t-2}}\right\}$ be the real basis for $\left(\mathfrak{g} / \mathfrak{a}_{t-2}\right)^{*}$ dual to the basis $\left\{X_{1}, \bar{X}_{1}, \ldots, X_{n-n_{t-2}}, \bar{X}_{n-n_{t-2}}\right\}$ for $\mathfrak{g} / \mathfrak{a}_{t-2}$. Now, because the almost complex structure $J_{t-2}$ defined by (8) is integrable, conditions (11) and (12) imply

$$
\begin{aligned}
d \omega_{i}= & 0 \quad\left(1 \leq i \leq n-n_{t-1}\right), \\
d \omega_{k}= & \sum_{\substack{1 \leq i<j \leq n-n_{t-1}\\
}} A_{k i j} \omega_{i} \wedge \omega_{j}+\sum_{\substack{1 \leq i, j \leq n-n_{t-1}\\
}} B_{k i j} \omega_{i} \wedge \bar{\omega}_{j} \\
& \left(n-n_{t-1}+1 \leq k \leq n-n_{t-2}\right) .
\end{aligned}
$$

Equations (13) are of the same type as equations (2). Continuing with this procedure $t-2$ times on each step of the sequence (7), taking into account that $\mathfrak{a}_{l+1} / \mathfrak{a}_{l}$ is contained in the center of $\mathfrak{g} / \mathfrak{a}_{l}$ and that $J_{l}$ given by (8) is integrable, we obtain a real basis $\left\{\omega_{i}, \bar{\omega}_{i} ; 1 \leq i \leq n\right\}$ for $\mathfrak{g}^{*}$ such that equations $(2)$ are satisfied.

A well known result of $\mathrm{Mal}^{\prime} \mathrm{cev} \mathrm{Ma}$ implies that a simply-connected connected nilpotent Lie group $G$ has compact quotients of the form $\Gamma \backslash G, \Gamma$ being a lattice in $G$, provided there exists a basis of left invariant 1 -forms such that the coefficients in the structure equations are rational numbers. Now, from this result and Theorem 12 we have 
Theorem 13. The structure equations (2) define a simply-connected connected nilpotent Lie group $G$ with nilpotent (left invariant) complex structure; hence, there exist compact nilmanifolds $\Gamma \backslash G$ with nilpotent complex structures in the sense of Definition 8 (ii). Conversely, the structure equations for the Lie algebra $\mathfrak{g}$ of a nilpotent Lie group $G$ with a nilpotent (left invariant) complex structure have the form (2).

Remark 14. We note that Theorem 13 implies that Corollary 7 in [CFG4] must be corrected accordingly; that is, it applies only to nilpotent Lie groups $G$ with nilpotent (left invariant) complex structure.

Next, we show a necessary topological condition for a compact nilmanifold to have a nilpotent complex structure.

Proposition 15. Let $M=\Gamma \backslash G$ be a compact nilmanifold with a nilpotent complex structure. Then, the first Betti number of $M$ satisfies $b_{1}(M) \geq 3$.

Proof. By Nomizu's theorem [No] we have that $H^{1}(\Gamma \backslash G) \cong H^{1}(\mathfrak{g})$, where $H^{1}(\Gamma \backslash G)$ is the first de Rham cohomology group of $\Gamma \backslash G$, and $\mathfrak{g}$ denotes the Lie algebra of $G$. Since $\operatorname{dim} H^{1}(\mathfrak{g})=\operatorname{dim}(\mathfrak{g} /[\mathfrak{g}, \mathfrak{g}])$, from Proposition 11 we have that $\operatorname{dim} H^{1}(\Gamma \backslash G)=$ $\operatorname{dim} H^{1}(\mathfrak{g}) \geq 2 n-(2 n-3)=3$; that is, $b_{1}(\Gamma \backslash G) \geq 3$.

\section{Compact nilmanifolds With nilpotent Complex structure AND HOLOMORPHIC PRINCIPAL FIBRE BUNDLES}

In this section we describe how a compact nilmanifold with a nilpotent complex structure can be realized as the total space at the top in a tower of holomorphic principal bundles whose structure groups are all complex tori. We shall prove also that each bundle in this tower carries a canonical (compatible) principal connection.

Let $G$ be a simply-connected connected nilpotent Lie group with a nilpotent (left invariant) complex structure, $\mathfrak{g}$ its Lie algebra, $\left\{\mathfrak{a}_{l} ; l \geq 0\right\}$ the ascending series compatible with the complex structure of $G$, and (7) the induced sequence of Lie algebras and homomorphisms.

Let $\left\{\omega_{1}, \bar{\omega}_{1}, \ldots, \omega_{n}, \bar{\omega}_{n}\right\}$ be a real basis of $\mathfrak{g}^{*}$ satisfying the structure equations (2), and let $\left\{X_{1}, \bar{X}_{1}, \ldots, X_{n}, \bar{X}_{n}\right\}$ be the real basis of $\mathfrak{g}$ dual to this basis of 1-forms. Without loss of generality, we can assume that the basis $\left\{X_{i}, \bar{X}_{i} ; 1 \leq\right.$ $i \leq n\}$ is such that $\left\{X_{n-n_{l}+1}, \bar{X}_{n-n_{l}+1}, \ldots, X_{n}, \bar{X}_{n}\right\}$ is a real basis of $\mathfrak{a}_{l}, n_{l}=$ $\operatorname{dim}_{\mathbb{C}} \mathfrak{a}_{l}$. In fact, proceeding as in the proof of Theorem 12, having chosen a basis $\left\{X_{1}, \bar{X}_{1}, \ldots, X_{n-n_{t-1}}, \bar{X}_{n-n_{t-1}}\right\}$ of the Lie algebra $\mathfrak{g} / \mathfrak{a}_{t-1}$, we complete it to a basis $\left\{X_{1}, \bar{X}_{1}, \ldots, X_{n-n_{t-1}}, \bar{X}_{n-n_{t-1}}, X_{n-n_{t-1}+1}, \bar{X}_{n-n_{t-1}+1}, \ldots, X_{n-n_{t-2}}, \bar{X}_{n-n_{t-2}}\right\}$ of the Lie algebra $\mathfrak{g} / \mathfrak{a}_{t-2}$, and so on, until we have a basis $\left\{X_{1}, \bar{X}_{1}, \ldots, X_{n}, \bar{X}_{n}\right\}$ of the Lie algebra $\mathfrak{g}$ (see Example 4 in Section 5). Thus, $\left\{X_{n-n_{l}+1}, \bar{X}_{n-n_{l}+1}, \ldots\right.$, $\left.X_{n}, \bar{X}_{n}\right\}$ is a basis for $\mathfrak{a}_{l}$, and $\left\{\omega_{i}, \bar{\omega}_{i} ; 1 \leq i \leq n-n_{l}\right\}$ determines the quotient Lie algebra $\mathfrak{g} / \mathfrak{a}_{l}$. Moreover, since $\left[\mathfrak{a}_{l+1}, \mathfrak{g}\right] \subset \mathfrak{a}_{l}$, we have

$$
\left[\mathfrak{a}_{l+1} / \mathfrak{a}_{l}, \mathfrak{a}_{l+1} / \mathfrak{a}_{l}\right] \subset\left[\mathfrak{a}_{l+1} / \mathfrak{a}_{l}, \mathfrak{g} / \mathfrak{a}_{l}\right]=0 .
$$

This implies that the coefficients $A_{i j k}$ and $B_{i j k}$ are zero if $j>n-n_{l+1}$ or if $k>n-n_{l+1}$; that is, the structure equations for $\mathfrak{g} / \mathfrak{a}_{l}$ are

$$
d \omega_{i}=\sum_{j<k \leq n-n_{l+1}} A_{i j k} \omega_{j} \wedge \omega_{k}+\sum_{j, k \leq n-n_{l+1}} B_{i j k} \omega_{j} \wedge \bar{\omega}_{k} \quad\left(1 \leq i \leq n-n_{l}\right) .
$$


(Here we are denoting with the same symbol $\omega_{i}$ the element in $\mathfrak{g}$ and the one induced on the quotient.) Therefore, $\mathfrak{g} / \mathfrak{a}_{l}$ is itself the Lie algebra of a simply-connected connected nilpotent Lie group $G_{l}$ with a nilpotent (left invariant) complex structure, which is exactly the almost complex structure $J_{l}$ defined by (8); hence $\operatorname{dim}_{\mathbb{C}} G_{l}=$ $n-n_{l}$. Moreover, $\operatorname{ker} \pi_{l}=\mathfrak{a}_{l} / \mathfrak{a}_{l-1}$ is a complex Abelian Lie algebra of complex dimension $n_{l}-n_{l-1}$; therefore, it defines a simply-connected connected Abelian complex Lie group $A_{l-1}$, that is, $A_{l-1} \cong \mathbb{C}^{n_{l}-n_{l-1}}$. Furthermore, since $\mathfrak{a}_{l} / \mathfrak{a}_{l-1}$ is an ideal in $\mathfrak{g} / \mathfrak{a}_{l-1}, A_{l-1}$ is a closed normal Lie subgroup of $G_{l-1}$ and $G_{l}=G_{l-1} / A_{l-1}$. In particular, $\operatorname{ker} \pi_{t}=\mathfrak{a}_{t} / \mathfrak{a}_{t-1}=\mathfrak{g} / \mathfrak{a}_{t-1}$ is also Abelian and it defines the complex Lie group $\mathbb{C}^{n-n_{t-1}}$. A basis of 1 -forms for $\mathfrak{a}_{l} / \mathfrak{a}_{l-1}$ is obtained by considering the 1 -forms $\omega_{i}, \bar{\omega}_{i}, n-n_{l}+1 \leq i \leq n-n_{l-1}$, as closed forms.

Integrating the Lie algebra homomorphisms in (7), we get a tower of Lie groups and surjective homomorphisms $\pi_{l}$ with Abelian kernels

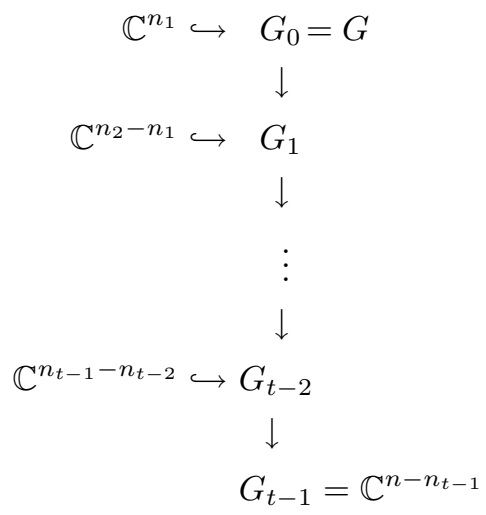

where each $\pi_{l}: G_{l-1} \rightarrow G_{l}$ is holomorphic because it is obtained by integration of $\pi_{l}: \mathfrak{g} / \mathfrak{a}_{l-1} \rightarrow \mathfrak{g} / \mathfrak{a}_{l}$, which commutes with the (integrable) almost complex structures $J_{l-1}$ of $\mathfrak{g} / \mathfrak{a}_{l-1}$ and $J_{l}$ of $\mathfrak{g} / \mathfrak{a}_{l}$, respectively. Moreover, since $\left[\mathfrak{a}_{l} / \mathfrak{a}_{l-1}, \mathfrak{g} / \mathfrak{a}_{l-1}\right]=$ 0 we have an induced holomorphic free action of $\mathbb{C}^{n_{l}-n_{l-1}}$ on $G_{l-1}$. Thus, any simply-connected connected nilpotent Lie group $G$ with a nilpotent (left invariant) complex structure is realized as the total space at the top of a tower of holomorphic principal bundles with Abelian structure groups.

Suppose $\Gamma_{l-1}$ is a lattice in $G_{l-1}$. Since $A_{l-1}$ is contained in the center of $G_{l-1}$, $A_{l-1} \cap \Gamma_{l-1}$ is a lattice in $A_{l-1}$, and thus the compact nilmanifold

$$
\left(A_{l-1} \cap \Gamma_{l-1}\right) \backslash A_{l-1}
$$

is, up to isomorphism, the complex torus $\mathbb{T}^{n_{l}-n_{l-1}}$. Moreover, $A_{l-1} \Gamma_{l-1}$ is closed in $G_{l-1}$ and $\pi_{l}\left(\Gamma_{l-1}\right)$ is a lattice in $G_{l}\left[\underline{\mathrm{R}}\right.$. Let $\Gamma_{l}=\pi_{l}\left(\Gamma_{l-1}\right)$. Since

$$
\left(A_{l-1} \cap \Gamma_{l-1}\right) \backslash A_{l-1} \cong \Gamma_{l-1} \backslash A_{l-1} \Gamma_{l-1},
$$

we obtain the following holomorphic principal bundle:

$$
\mathbb{T}^{n_{l}-n_{l-1}} \hookrightarrow \Gamma_{l-1} \backslash G_{l-1} \stackrel{\pi_{l}}{\longrightarrow} \Gamma_{l} \backslash G_{l} .
$$

Let $\Gamma$ be a lattice in $G$. Then, taking into account the argument above, $\Gamma_{1}=\pi_{1}(\Gamma)$, $\Gamma_{2}=\pi_{2}\left(\Gamma_{1}\right), \ldots, \Gamma_{t-1}=\pi_{t-1}\left(\Gamma_{t-2}\right)$ are lattices in $G_{1}, G_{2}, \ldots, G_{t-1}$, respectively. Therefore, replacing $G$ by $\Gamma \backslash G$ and $G_{l}$ by $\Gamma_{l} \backslash G_{l}(1 \leq l \leq t-1)$ in the tower above, 
we get a new tower of holomorphic principal bundles

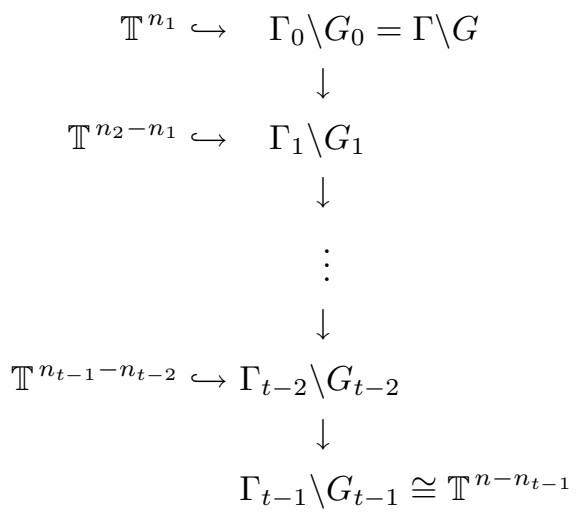

where $\mathbb{T}^{p}$ denotes the complex torus of complex dimension $p$. Thus the compact nilmanifold $\Gamma \backslash G$ with a nilpotent complex structure is realized as the total space at the top of a tower of holomorphic principal bundles with structure groups complex tori. We note that each manifold $\Gamma_{l} \backslash G_{l}$ in this tower is itself a compact nilmanifold with nilpotent complex structure.

It is worthwhile to compare this construction with the constructions and results of S. Murakami [Mu which are concerned with compact complex nilmanifolds. In that paper, Murakami proved that the total space of a holomorphic principal bundle over a complex torus having connected Abelian structure group is a compact complex nilmanifold. Murakami's proof of this assertion makes use of the existence of a canonical principal connection on the bundle.

Here, at each step (14) of the tower of bundles, there exists also a canonical principal connection, defined as follows. First, note that the basis of left invariant 1 -forms $\left\{\omega_{1}, \ldots, \omega_{n-n_{l-1}}\right\}$ of type $(1,0)$ on $G_{l-1}$ descends to the quotient manifold $\Gamma_{l-1} \backslash G_{l-1}$ with nilpotent complex structure; we shall denote the forms on the quotient manifold by the same symbols. Let $\left\{Z_{1}, \ldots, Z_{n_{l}-n_{l-1}}\right\}$ be a basis of left invariant holomorphic vector fields of type $(1,0)$ on the structure group $\mathbb{T}^{n_{l}-n_{l-1}}$; then

$$
\boldsymbol{\omega}=\sum_{i=1}^{n_{l}-n_{l-1}} \omega_{n-n_{l}+i} \otimes Z_{i}
$$

defines a principal connection on the bundle; this connection form has type $(1,0)$ and is compatible with the holomorphic structures $[\mathrm{A}], \mathrm{KS}$. Since the structure group is Abelian, we have $d \boldsymbol{\omega}=\pi_{l}^{*} \boldsymbol{\Omega}$, where $\boldsymbol{\Omega}$ denotes the curvature form of $\boldsymbol{\omega}$ on the base $\Gamma_{l} \backslash G_{l}$, which is given by

$$
\begin{aligned}
\mathbf{\Omega}=\sum_{i=1}^{n_{l}-n_{l-1}}\left(\sum_{j<k \leq n-n_{l}} A_{n-n_{l}+i j k} \omega_{j} \wedge \omega_{k}\right. \\
\left.+\sum_{j, k \leq n-n_{l}} B_{n-n_{l}+i j k} \omega_{j} \wedge \bar{\omega}_{k}\right) \otimes Z_{i} .
\end{aligned}
$$

Now, since the structure group of this bundle is Abelian, then its adjoint bundle is the product bundle $\left(\Gamma_{l} \backslash G_{l}\right) \times \mathbb{C}^{n_{l}-n_{l-1}}$; this allows us to identify the differential 
forms on $\Gamma_{l} \backslash G_{l}$ with values in the adjoint bundle to $\mathbb{C}^{n_{l}-n_{l-1}}$-valued differential forms. Thus, the component of type $(1,1)$ of the curvature form,

$$
\Omega^{1,1}=\sum_{i=1}^{n_{l}-n_{l-1}}\left(\sum_{j, k \leq n-n_{l}} B_{n-n_{l}+i j k} \omega_{j} \wedge \bar{\omega}_{k}\right) \otimes Z_{i},
$$

defines a cohomology class $\left[\boldsymbol{\Omega}^{1,1}\right] \in H_{\bar{\partial}}^{1,1}\left(\Gamma_{l} \backslash G_{l}, \mathbb{C}^{n_{l}-n_{l-1}}\right)$, which is known to be independent of the choice of $\boldsymbol{\omega}$ and is called the Atiyah class of the bundle $\mathrm{A}$, $\mathrm{KS}$. Therefore, if some coefficient $B_{i j k}$ in (18) is nonzero, the Atiyah class of the bundle (14) does not vanish or, which is equivalent, the bundle does not admit holomorphic connections. If $\Gamma \backslash G$ is a compact complex parallelizable nilmanifold, that is, if the coefficients $B_{i j k}$ in (2) are all zero, then all the bundles in the tower (15) have vanishing Atiyah class; indeed, $\boldsymbol{\omega}$ in (16) is a holomorphic connection. The examples in Section 5 will illustrate both possibilities.

Remark 16. If $\Gamma \backslash G$ is assumed to be a compact (real) nilmanifold, then the ascending series $\left\{\mathfrak{a}_{l}\right\}$ is not defined. But a similar construction for $\Gamma \backslash G$ can be done by using the ascending central series $\left\{\mathfrak{g}_{l}\right\}$ instead of $\left\{\mathfrak{a}_{l}\right\}$. Thus, $\Gamma \backslash G$ is realized as the total space at the top of a tower of differentiable principal bundles with structure groups real tori. Moreover, canonical principal connections can be defined at each step of the tower in a similar way (see [CFG5] for the details).

Remark 17. If $\Gamma \backslash G$ is assumed to be a compact complex parallelizable nilmanifold in the sense of Wang Wa], then $\mathfrak{g}$ is a complex Lie algebra and $\mathfrak{g}_{l}$ is a complex Lie subalgebra of $\mathfrak{g}$; in fact $\mathfrak{a}_{l}=\mathfrak{g}_{l}$ for all $l$. Therefore, a similar construction for $\Gamma \backslash G$ can be done, so that $\Gamma \backslash G$ is realized as the total space at the top of a tower of holomorphic principal bundles whose structure groups are complex tori. As mentioned above, the connection $\boldsymbol{\omega}$ will be holomorphic in this case.

\section{Minimal model for the Dolbeault Cohomology OF A COMPACT NILMANIFOLD WITH NILPOTENT COMPLEX STRUCTURE}

4.1. Basics of Dolbeault homotopy theory. A Dolbeault homotopy theory has been developed in [NT by defining "complex homotopy groups" in terms of the Dolbeault complex of the manifold. The following are basic definitions of this theory.

A differential bigraded algebra $\mathcal{A}^{*, *}$ is a bigraded commutative algebra over $\mathbb{C}$ with a differential $\bar{\partial}$ of type $(0,1)$ which is a derivation, i.e. $\bar{\partial}(a b)=(\bar{\partial} a) b+$ $(-1)^{\operatorname{deg} a} a(\bar{\partial} b)$, where $\operatorname{deg} a$ is the total degree of $a$. It is further required that $\mathcal{A}$ be augmented over $\mathbb{C}$. Morphisms between differential bigraded algebras are required to be bidegree preserving algebra maps which commute with the differentials.

The most immediate example of a differential bigraded algebra is the Dolbeault complex $\left(\Lambda_{\mathbb{C}}^{*, *} M, \bar{\partial}\right)$ of a complex manifold $M$; the algebra $\Lambda_{\mathbb{C}}^{*, *} M$ is augmented by choosing a point $p \in M$ and evaluating the $C^{\infty}$ complex valued functions at $p$. Since these functions are $\Lambda_{\mathbb{C}}^{0,0} M$, this augments $\Lambda_{\mathbb{C}}^{*, *} M$. Note that $\Lambda_{\mathbb{C}}^{*, *} M$ as an augmented algebra depends on the base point. If $M$ has no nonconstant holomorphic functions, for example if $M$ is compact connected, then $H_{\bar{\partial}}^{0,0}(M)=\mathbb{C}$ and the dependence on the base point, at the cohomology level, is only up to isomorphism.

Another immediate example is the following. Let $\mathfrak{h}$ be a complex Lie algebra, and let $\mathfrak{h}^{*}$ be its dual. Let us consider their complexifications endowed with their 
natural decompositions:

$$
\mathfrak{h}^{\mathbb{C}}=\mathfrak{h}_{1,0}+\mathfrak{h}_{0,1}, \quad\left(\mathfrak{h}^{*}\right)^{\mathbb{C}}=\mathfrak{h}^{1,0}+\mathfrak{h}^{0,1} .
$$

Since $\left(\mathfrak{h}^{\mathbb{C}}\right)^{*} \cong\left(\mathfrak{h}^{*}\right)^{\mathbb{C}}$, there is a canonical structure of bigraded commutative algebra in the exterior algebra $\Lambda^{*}\left(\mathfrak{h}^{\mathbb{C}}\right)^{*}$ :

$$
\Lambda^{*}\left(\mathfrak{h}^{\mathbb{C}}\right)^{*}=\bigoplus_{p, q \geq 0} \Lambda^{p, q}\left(\mathfrak{h}^{\mathbb{C}}\right)^{*}
$$

where $\Lambda^{p, q}\left(\mathfrak{h}^{\mathbb{C}}\right)^{*}=\left(\Lambda^{p} \mathfrak{h}^{1,0}\right) \otimes\left(\Lambda^{q} \mathfrak{h}^{0,1}\right)$. Consider the Chevalley-Eilenberg differential $d: \Lambda^{*} \mathfrak{h}^{*} \longrightarrow \Lambda^{*+1} \mathfrak{h}^{*}$ given by

$$
(d \alpha)\left(x_{1}, \ldots, x_{k+1}\right)=\sum_{i<j}(-1)^{i+j} \alpha\left(\left[x_{i}, x_{j}\right], x_{1}, \ldots, \widehat{x_{i}}, \ldots, \widehat{x_{j}}, \ldots, x_{k+1}\right),
$$

where $\alpha \in \Lambda^{k} \mathfrak{h}^{*}, x_{1}, \ldots, x_{k+1} \in \mathfrak{h}$; then $d$ extends in a natural way to $\Lambda^{*}\left(\mathfrak{h}^{\mathbb{C}}\right)^{*}$. Since $\mathfrak{h}$ is a complex Lie algebra,

$$
\left[\mathfrak{h}_{1,0}, \mathfrak{h}_{1,0}\right] \subset \mathfrak{h}_{1,0}, \quad\left[\mathfrak{h}_{0,1}, \mathfrak{h}_{0,1}\right] \subset \mathfrak{h}_{0,1}, \quad\left[\mathfrak{h}_{1,0}, \mathfrak{h}_{0,1}\right]=0,
$$

and therefore $d$ decomposes as $d=\partial+\bar{\partial}$, where

$$
\partial: \Lambda^{p, q}\left(\mathfrak{h}^{\mathbb{C}}\right)^{*} \longrightarrow \Lambda^{p+1, q}\left(\mathfrak{h}^{\mathbb{C}}\right)^{*}, \quad \bar{\partial}: \Lambda^{p, q}\left(\mathfrak{h}^{\mathbb{C}}\right)^{*} \longrightarrow \Lambda^{p, q+1}\left(\mathfrak{h}^{\mathbb{C}}\right)^{*},
$$

and $\partial^{2}=\partial \bar{\partial}+\bar{\partial} \partial=\bar{\partial}^{2}=0$. Thus $\left(\Lambda^{* * *}\left(\mathfrak{h}^{\mathbb{C}}\right)^{*}, \bar{\partial}\right)$ is a differential bigraded algebra. This construction also applies to the Lie algebra $\mathfrak{g}$ of a Lie group $G$ with a left invariant integrable almost complex structure (however, in this case $\left[\mathfrak{g}_{1,0}, \mathfrak{g}_{0,1}\right]$ can be nonzero); then $\left(\Lambda^{* * *}\left(\mathfrak{g}^{\mathbb{C}}\right)^{*}, \bar{\partial}\right)$ is canonically identified to the differential bigraded algebra of the complex valued left invariant differential forms on $G$.

Given a differential bigraded algebra $\left(\mathcal{A}^{*, *}, \bar{\partial}\right)$, we shall say that it is a model for the Dolbeault cohomology of a complex manifold $M$ if there exists a morphism of differential bigraded algebras $\rho: \mathcal{A}^{*, *} \rightarrow \Lambda_{\mathbb{C}}^{*, *} M$ inducing an isomorphism on cohomology. A model $\left(\mathcal{A}^{*, *}, \bar{\partial}\right)$ is said to be minimal if: (a) $\mathcal{A}^{*, *}$ is free as an algebra; (b) there exists a collection of generators $\left\{a_{\tau}\right\}_{\tau \in I}$, for some well ordered index set $I$, such that each $\bar{\partial}\left(a_{\tau}\right)$ is expressed in terms of preceding generators $a_{\mu}$ $(\mu<\tau)$ of total degree $\operatorname{deg}\left(a_{\mu}\right) \leq \operatorname{deg}\left(a_{\tau}\right)$. A model $\left(\mathcal{A}^{*, *}, \bar{\partial}\right)$ is said to be formal if there is a morphism of differential bigraded algebras $\psi:\left(\mathcal{A}^{*, *}, \bar{\partial}\right) \rightarrow\left(H_{\bar{\partial}}^{*, *}(\mathcal{A}), \bar{\partial}=\right.$ 0 ) inducing the identity on cohomology.

Massey (triple) products can be defined for $H_{\bar{\partial}}^{*, *}(\mathcal{A})$ in the standard form. Let $\boldsymbol{\alpha} \in H_{\bar{\partial}}^{p, q}(\mathcal{A}), \boldsymbol{\beta} \in H_{\bar{\partial}}^{r, s}(\mathcal{A}), \boldsymbol{\gamma} \in H_{\bar{\partial}}^{u, v}(\mathcal{A})$ satisfy $\boldsymbol{\alpha} \cdot \boldsymbol{\beta}=0=\boldsymbol{\beta} \cdot \boldsymbol{\gamma}$. Let $\alpha, \beta$ and $\gamma$ be $\bar{\partial}$-closed forms representing $\boldsymbol{\alpha}, \boldsymbol{\beta}$ and $\boldsymbol{\gamma}$; then $\alpha \wedge \beta=\bar{\partial} \eta, \beta \wedge \gamma=\bar{\partial} \mu$, and so $\eta \wedge \gamma+(-1)^{p+q+1} \alpha \wedge \mu$ is a $\bar{\partial}$-closed form of type $(p+r+u, q+s+v-1)$ whose cohomology class is well defined modulo the ideal $I=\boldsymbol{\alpha} \cdot H_{\bar{\partial}}^{r+u, s+v-1}(\mathcal{A})+$ $\boldsymbol{\gamma} \cdot H_{\bar{\partial}}^{p+r, q+s-1}(\mathcal{A})$. This class in $H_{\bar{\partial}}^{p+r+u, q+s+v-1}(\mathcal{A}) / I$ will be represented as $\langle\boldsymbol{\alpha}, \boldsymbol{\beta}, \boldsymbol{\gamma}\rangle$ and called the Massey (triple) product of $\boldsymbol{\alpha}, \boldsymbol{\beta}$ and $\boldsymbol{\gamma}$. As it happens for the de Rham homotopy theory (see [DGMS], GM]), nonzero Massey products are obstructions to the formality of a model $\left(\mathcal{A}^{*, *}, \bar{\partial}\right)$.

4.2. Hirsch Lemma for Dolbeault cohomology. In [Hi Appendix Two], A. Borel defined a spectral sequence associated to a complex analytic bundle with compact connected fibres. This spectral sequence relates the Dolbeault cohomology groups of the total space, of the base space and of the typical fibre of the bundle. 
Let us recall briefly Borel's results for the particular case of a (not necessarily principal) holomorphic fibre bundle $F \hookrightarrow E \stackrel{\pi}{\longrightarrow} B$, where $F, E$ and $B$ are assumed to be compact connected complex manifolds and the structure group of the fibration is connected. For such a holomorphic fibration, Borel's results can be read as follows: there exists a spectral sequence $\left(E_{r}, d_{r}\right), r \geq 0$, such that:

1. $E_{r}$ is 4 -graded, by the base-degree, the fibre-degree and the type. Let ${ }^{p, q} E_{r}^{s, t}$ be the subspace of elements of $E_{r}$ of (total) type $(p, q)$, base-degree $s$, fibredegree $t$. Then:

(a) ${ }^{p, q} E_{r}^{s, t}=0$ if $p+q \neq s+t$ or if one of $p, q, s, t$ is $<0$;

(b) $d_{r}:{ }^{p, q} E_{r}^{s, t} \longrightarrow{ }^{p, q+1} E_{r}^{s+r, t-r+1}$.

2. If $p+q=s+t$, then

$$
{ }^{p, q} E_{2}^{s, t} \cong \sum_{i \geq 0} H_{\bar{\partial}}^{i, s-i}(B) \otimes H_{\bar{\partial}}^{p-i, q-s+i}(F)
$$

3. The spectral sequence converges to $H_{\bar{\partial}}^{*, *}(E)$, the Dolbeault cohomology of the total space. For all $p, q \geq 0$,

$$
\operatorname{Gr} H_{\bar{\partial}}^{p, q}(E)=\sum_{s+t=p+q}{ }^{p, q} E_{\infty}^{s, t}
$$

for a suitable filtration of $H_{\bar{\partial}}^{p, q}(E)$.

4. $\left(E_{r}, d_{r}\right)$ consists of differential commutative algebras, and the isomorphism in (19) is compatible with the product.

Let us recall also that Borel's filtration is given by the submodules

$$
L^{k}\left(\Lambda_{\mathbb{C}}^{*} E\right)=\sum_{a+b \geq k} M^{a, b, c, d},
$$

where the elements in $M^{a, b, c, d}$ are to be thought of as $(a, b)$-forms on $B$ with coefficients in $(c, d)$-forms in the fibre. The total bidegree $(p, q)$ is determined by $p=a+c, q=b+d$.

We shall use Borel's results to prove a Hirsch Lemma that will allow the determination of a model for the Dolbeault cohomology of the total space of a holomorphic fibration satisfying some suitable hypothesis.

Let $F \hookrightarrow E \stackrel{\pi}{\longrightarrow} B$ be a holomorphic fibration, where $E, B, F$ are connected, $F$ is compact and the structure group of the fibration is connected. An element $\boldsymbol{\alpha} \in H_{\bar{\partial}}^{p, q}(F)$ is said to be transgressive if there exists a representative $\alpha \in \Lambda_{\mathbb{C}}^{p, q} F$ which extends to a form $\tilde{\alpha} \in \Lambda_{\mathbb{C}}^{p, q} E$ such that $\bar{\partial} \tilde{\alpha}=\pi^{*} \beta$ for some $\bar{\partial}$-closed form $\beta \in \Lambda_{\mathbb{C}}^{p, q+1} B$. If $H_{\bar{\partial}}^{*, *}(F)$ is free as a bigraded algebra, we say that it is transgressive if it has an algebra basis consisting of transgressive elements.

Assume that the holomorphic fibration has $H_{\bar{\partial}}^{*, *}(F)$ free and transgressive. Let $\left(\mathcal{A}^{*, *}, \bar{\partial}\right)$ be a differential bigraded algebra and $\rho: \mathcal{A}^{*, *} \rightarrow \Lambda_{\mathbb{C}}^{*, *} B$ a morphism of differential bigraded algebras giving an isomorphism on cohomology; that is, $\left(\mathcal{A}^{*, *}, \bar{\partial}\right)$ is a model for $\left(\Lambda_{\mathbb{C}}^{*, *} B, \bar{\partial}\right)$. Pick an algebra basis $\left\{\boldsymbol{x}_{1}, \ldots, \boldsymbol{x}_{p}\right\}$ for $H_{\bar{\partial}}^{*, *}(F)$. Let $\tilde{\alpha}_{i} \in \Lambda_{\mathbb{C}}^{*, *} E$ be a form which when restricted to $F$ gives a $\bar{\partial}$-closed form representing $\boldsymbol{x}_{i}$. Let $\beta_{i}$ be such that $\bar{\partial} \tilde{\alpha}_{i}=\pi^{*}\left(\beta_{i}\right)$. Since $\rho$ is an isomorphism on cohomology, we may pick $a_{i}$ such that $\beta_{i}=\rho\left(a_{i}\right)$ for some $\bar{\partial}$-closed form $a_{i} \in \mathcal{A}^{* * *}$. Let $T=\mathcal{A}^{*, *} \otimes H_{\bar{\partial}}^{*, *}(F)$ be the tensor product of these bigraded algebras, and define 
a differential $\bar{\partial}$ of type $(0,1)$ for $T$ by setting $\bar{\partial}: H_{\bar{\partial}}^{*, *}(F) \rightarrow \mathcal{A}^{*, *+1}$ by $\bar{\partial}\left(\boldsymbol{x}_{i}\right)=a_{i}$. Then $(T, \bar{\partial})$ is a differential bigraded algebra. Define

$$
\tilde{\rho}: T=\mathcal{A}^{*, *} \otimes H_{\bar{\partial}}^{*, *}(F) \longrightarrow \Lambda_{\mathbb{C}}^{*, *} E
$$

by $\tilde{\rho}_{\mid \mathcal{A}}=\pi^{*} \circ \rho, \tilde{\rho}\left(\boldsymbol{x}_{i}\right)=\tilde{\alpha}_{i}$.

Lemma 18 (Hirsch Lemma [Co]). The morphism $\tilde{\rho}$ in (21) induces an isomorphism on cohomology. Hence, $\left(\mathcal{A}^{*, *} \otimes H_{\bar{\partial}}^{*, *}(F), \bar{\partial}\right)$ is a model for the Dolbeault complex $\left(\Lambda_{\mathbb{C}}^{*, *} E, \bar{\partial}\right)$.

Proof. We shall construct a spectral sequence $\hat{E}_{r}$ converging to the cohomology $H_{\bar{\partial}}^{* * *}(T)$, and such that $\tilde{\rho}$ induces an isomorphism of $\hat{E}_{2}$ into the $E_{2}$-terms in Borel's spectral sequence; then the result will follow from Borel's in Hi]. First, let us consider the particular case of $\mathcal{A}^{*, *}=\Lambda_{\mathbb{C}}^{*, *} B$ and $\rho=$ identity. Put

$$
T=\bigoplus_{p, q}^{p, q} T
$$

with

$$
{ }^{p, q} T=\sum_{\substack{a+c=p \\ b+d=q}} \Lambda_{\mathbb{C}}^{a, b} B \otimes H_{\bar{\partial}}^{c, d}(F)
$$

and define

$$
L^{k} T=\sum_{a+b \geq k} \Lambda_{\mathbb{C}}^{a, b} B \otimes H_{\bar{\partial}}^{c, d}(F)
$$

then

$$
\begin{aligned}
L^{0} T=T, & L^{k} T=0 \text { if } k>\operatorname{dim}_{\mathbb{R}} B, \\
L^{k} T \supset L^{k+1} T, & \bar{\partial}\left(L^{k} T\right) \subset L^{k} T,
\end{aligned}
$$

and, therefore, $\left\{L^{k} T\right\}$ is a bounded decreasing filtration of $T$ which is stable under $\bar{\partial}$; hence, we may consider the associated spectral sequence $\left(\hat{E}_{r}, d_{r}\right), r \geq 0$. If we set ${ }^{p, q} L^{k} T=\left(L^{k} T\right) \cap{ }^{p, q} T$, then $L^{k} T=\sum_{p, q \geq 0}{ }^{p, q} L^{k} T$ and, since $\bar{\partial}$ is homogeneous of degree 1 in $q$ and of degree 0 in $p$, this bigrading $(p, q)$ is also present in the terms $\hat{E}_{r}$. Let ${ }^{p, q} \hat{E}_{r}^{s, t}$ denote the terms of $\hat{E}_{r}$ of type $(p, q)$, total degree $s+t$ and degree $s$ in the grading defined by the filtration; that is, ${ }^{p, q} \hat{E}_{r}^{s, t}=0$ if $p+q \neq s+t$, and

$$
{ }^{p, q} \hat{E}_{r}^{s, t}=\frac{{ }^{p, q} Z_{r}^{s, t}}{{ }^{p, q} Z_{r-1}^{s+1, t-1}+{ }^{p, q} B_{r-1}^{s, t}},
$$

where

$$
\begin{aligned}
& { }^{p, q} Z_{r}^{s, t}={ }^{p, q} L^{s} T^{s+t} \cap \bar{\partial}^{-1}\left({ }^{p, q+1} L^{s+r} T^{s+t+1}\right), \\
& { }^{p, q} B_{r}^{s, t}={ }^{p, q} L^{s} T^{s+t} \cap \bar{\partial}\left({ }^{p, q-1} L^{s-r} T^{s+t-1}\right),
\end{aligned}
$$

and $d_{r}:{ }^{p, q} \hat{E}_{r}^{s, t} \longrightarrow{ }^{p, q+1} \hat{E}_{r}^{s+r, t-r+1}$. From the standard theory of spectral sequences, it follows easily that this spectral sequence converges to $H_{\bar{\partial}}^{*, *}(T)$.

Now, we note that $\tilde{\rho}$ in (21) is filtration preserving with respect to the Borel filtration of $\Lambda_{\mathbb{C}}^{* * *} E$. In fact, let

$$
\omega_{k}=\sum_{a+b \geq k} \omega^{a, b} \otimes \boldsymbol{x}^{c, d} \in L^{k} T, \quad \omega^{a, b} \in \Lambda_{\mathbb{C}}^{a, b} B, \quad x^{c, d} \in H_{\bar{\partial}}^{c, d}(F),
$$


and let $\tilde{\alpha}^{c, d} \in \Lambda_{\mathbb{C}}^{c, d} E$ restrict to a representative of $\boldsymbol{x}^{c, d}$. Therefore $\tilde{\rho}\left(\omega_{k}\right)=$ $\sum_{a+b \geq k} \pi^{*}\left(\omega^{a, b}\right) \wedge \tilde{\alpha}^{c, d} \in L^{k}\left(\Lambda_{\mathbb{C}}^{*} E\right)$, where $L^{k}\left(\Lambda_{\mathbb{C}}^{*} E\right)$ is the module in Borel's filtration (20). Thus, $\tilde{\rho}$ induces a morphism of spectral sequences $\tilde{\rho}_{r}: \hat{E}_{r} \rightarrow E_{r}$ such that, indeed, $\tilde{\rho}_{2}: \hat{E}_{2} \rightarrow E_{2}$ is an isomorphism. In order to prove this assertion, suppose that $\left\{\boldsymbol{x}_{1}, \ldots, \boldsymbol{x}_{p}\right\}$ is a transgressive basis for $H_{\bar{\partial}}^{*, *}(F)$, and let $\tilde{\alpha}_{j} \in \Lambda_{\mathbb{C}}^{*, *} E$ restrict to a representative of $\boldsymbol{x}_{j}$ such that $\bar{\partial} \tilde{\alpha}_{j}=\pi^{*}\left(\beta_{j}\right)$, where $\beta_{j}$ is a $\bar{\partial}$-closed form on the base $B$, for $j=1, \ldots, p$. Then, each element $\boldsymbol{x}$ in $H_{\bar{\partial}}^{*, *}(F)$ can be expressed as a sum of products $\boldsymbol{x}_{r_{1}} \wedge \ldots \wedge \boldsymbol{x}_{r_{k}}$, with $1 \leq k \leq p$ and $\left\{r_{1}, \ldots, r_{k}\right\} \subseteq\{1, \ldots, p\}$. So, $\bar{\partial}\left(\boldsymbol{x}_{r_{1}} \wedge \ldots \wedge \boldsymbol{x}_{r_{k}}\right)$ is a sum of elements of the form

$$
\beta_{r_{j}} \otimes\left(\boldsymbol{x}_{r_{1}} \wedge \ldots \wedge \boldsymbol{x}_{r_{j-1}} \wedge \boldsymbol{x}_{r_{j+1}} \wedge \ldots \wedge \boldsymbol{x}_{r_{k}}\right)
$$

with filtration degree at least 2, because the total degree of a representative of $\boldsymbol{x}_{j}$ is at least 1 and $\beta_{j}=\bar{\partial}\left(\boldsymbol{x}_{j}\right), j=1, \ldots, p$. Therefore, if $\omega \otimes \boldsymbol{x}, \omega \in \Lambda_{\mathbb{C}}^{a, b} B$, has filtration degree $u=a+b$, then we have

$$
\bar{\partial}(\omega \otimes \boldsymbol{x})=\bar{\partial}(\omega) \otimes \boldsymbol{x}+(-1)^{u} \omega \otimes \bar{\partial}(\boldsymbol{x}),
$$

where $\bar{\partial}(\omega) \otimes \boldsymbol{x}$ has filtration degree $u+1$ and $\omega \otimes \bar{\partial}(\boldsymbol{x})$ has filtration degree at least $u+2$.

Now, we notice that

$$
{ }^{p, q} L^{s} T^{s+t}={ }^{p, q} Q^{s, t} \oplus{ }^{p, q} L^{s+1} T^{s+t},
$$

where

$$
{ }^{p, q} Q^{s, t}=\sum_{i \geq 0} \Lambda_{\mathbb{C}}^{i, s-i} B \otimes H_{\bar{\partial}}^{p-i, q-s+i}(F)
$$

therefore

$$
{ }^{p, q} Z_{2}^{s, t}=\left({ }^{p, q} Q^{s, t} \cap \bar{\partial}^{-1}\left({ }^{p, q+1} L^{s+2} T^{s+t+1}\right)\right) \oplus{ }^{p, q} Z_{1}^{s+1, t-1} .
$$

Now let

$$
\omega_{s}=\sum_{i \geq 0} \omega^{i, s-i} \otimes \boldsymbol{x}^{p-i, q-s+i} \in{ }^{p, q} Q^{s, t}
$$

then, it follows from $(22)$ that

$$
\bar{\partial}\left(\omega_{s}\right)=\sum_{i \geq 0}\left(\bar{\partial}\left(\omega^{i, s-i}\right) \otimes \boldsymbol{x}^{p-i, q-s+i}+(-1)^{s} \omega^{i, s-i} \otimes \bar{\partial}\left(\boldsymbol{x}^{p-i, q-s+i}\right)\right),
$$

where $\omega^{i, s-i} \otimes \bar{\partial}\left(\boldsymbol{x}^{p-i, q-s+i}\right)$ has filtration degree at least $s+2$, and therefore

$$
\bar{\partial}\left(\omega_{s}\right)=\sum_{i \geq 0} \bar{\partial}\left(\omega^{i, s-i}\right) \otimes \boldsymbol{x}^{p-i, q-s+i}+\text { terms in }{ }^{p, q+1} L^{s+2} T^{s+t+1} .
$$

Since the first summands in this expression all have filtration degree $s+1$, it follows that $\omega_{s} \in{ }^{p, q} Q^{s, t} \cap \bar{\partial}^{-1}\left({ }^{p, q+1} L^{s+2} T^{s+t+1}\right)$ if and only if each $\omega^{i, s-i}$ in (23) is $\bar{\partial}-$ closed.

On the other hand, an element $\omega_{s-1} \in{ }^{p, q-1} L^{s-1} T^{s+t-1}$ is of the form

$$
\omega_{s-1}=\sum_{i \geq 0} \omega^{i, s-i-1} \otimes \boldsymbol{x}^{p-i, q-s+i}+\sum_{k \geq s} \sum_{i \geq 0} \omega^{i, k-i} \otimes \boldsymbol{x}^{p-i, q-k-1+i},
$$

and therefore, using (22) again, we conclude that

$$
\bar{\partial}\left(\omega_{s-1}\right)=\sum_{i \geq 0} \bar{\partial}\left(\omega^{i, s-i-1}\right) \otimes \boldsymbol{x}^{p-i, q-s+i}+\text { terms in }^{p, q} Z_{1}^{s+1, t-1} .
$$


Then, it is obvious that

$$
{ }^{p, q} \hat{E}_{2}^{s, t} \cong \sum_{i \geq 0} H_{\bar{\partial}}^{i, s-i}(B) \otimes H_{\bar{\partial}}^{p-i, q-s+i}(F)
$$

and, hence, $\tilde{\rho}_{2}$ is an isomorphism. Therefore, $\tilde{\rho}$ also induces an isomorphism

$$
\tilde{\rho}: H_{\bar{\partial}}^{*, *}\left(\Lambda_{\mathbb{C}} B \otimes H_{\bar{\partial}}(F)\right) \stackrel{\cong}{\longrightarrow} H_{\bar{\partial}}^{*, *}(E) .
$$

Finally, a standard argument shows that

$$
\rho \otimes i d: \mathcal{A}^{*, *} \otimes H_{\bar{\partial}}^{*, *}(F) \longrightarrow \Lambda_{\mathbb{C}}^{*, *} B \otimes H_{\bar{\partial}}^{*, *}(F)
$$

is an isomorphism on cohomology if $\rho$ is.

The following corollaries are direct consequences of Lemma 18.

Corollary 19 (Künneth formula). Let $M, N$ be compact connected complex manifolds. Then

$$
H_{\bar{\partial}}^{*, *}(M \times N) \cong H_{\bar{\partial}}^{*, *}(M) \otimes H_{\bar{\partial}}^{*, *}(N)
$$

i.e.

$$
H_{\bar{\partial}}^{p, q}(M \times N) \cong \sum_{\substack{a+c=p \\ b+d=q}} H_{\bar{\partial}}^{a, b}(M) \otimes H_{\bar{\partial}}^{c, d}(N) .
$$

Corollary 20. Let $F \hookrightarrow E \stackrel{\pi}{\longrightarrow} B$ be a holomorphic fibration, $E, B, F$ being connected, $F$ compact and the structure group of the fibration also being connected. Assume that the fibration has $H_{\bar{\partial}}^{*, *}(F)$ free and transgressive. Denote $h^{p, q}()=$ $\operatorname{dim} H_{\bar{\partial}}^{p, q}()$. Then

$$
h^{p, q}(E) \leq \sum_{\substack{a+c=p \\ b+d=q}} h^{a, b}(B) \cdot h^{c, d}(F) .
$$

4.3. Proof of the Main Theorem. Suppose that $M=\Gamma \backslash G$ is a compact nilmanifold with a nilpotent complex structure, and so $G$ is defined by equations (2). Let (15) be its associated tower of holomorphic principal bundles. Consider one arbitrary step (14) of the tower. Let $\left\{\tau_{1}, \ldots, \tau_{n_{l}-n_{l-1}}\right\}$ be the basis of holomorphic 1-forms on the fibre $\mathbb{T}^{n_{l}-n_{l-1}}$ dual to the basis of vector fields $\left\{Z_{1}, \ldots, Z_{n_{l}-n_{l-1}}\right\}$. Since $\boldsymbol{\omega}$ in (16) is a connection, each 1 -form $\omega_{n-n_{l}+i}, 1 \leq$ $i \leq n_{l}-n_{l-1}$, on $\Gamma_{l-1} \backslash G_{l-1}$ restricts to $\tau_{i}$ on the fibre, and since $\Omega$ in (17) is the curvature of $\boldsymbol{\omega}$, it follows that each $\tau_{i}$ (resp. $\bar{\tau}_{i}$ ) transgresses to the $(1,1)$-form $\sum_{j, k \leq n-n_{l}} B_{n-n_{l}+i j k} \omega_{j} \wedge \bar{\omega}_{k}$ (resp. to the $(0,2)$-form $\sum_{j<k \leq n-n_{l}} \bar{A}_{n-n_{l}+i j k} \bar{\omega}_{j} \wedge$ $\left.\bar{\omega}_{k}\right)$ on $\Gamma_{l} \backslash G_{l}$. Therefore, $\left\{\tau_{i}, \bar{\tau}_{i}\right\}$ determines a transgressive basis for the Dolbeault cohomology $H_{\bar{\partial}}^{*, *}\left(\mathbb{T}^{n_{l}-n_{l-1}}\right)$ of the fibre.

Now, we start an iterative procedure from the bottom to the top of the tower. At the first step, the holomorphic principal bundle has $\mathbb{T}^{n-n_{t-1}}$ as the base and $\mathbb{T}^{n_{t-1}-n_{t-2}}$ as the fibre. Let us denote the minimal models for their Dolbeault cohomology as follows:

$$
\begin{aligned}
\mathbb{T}^{n-n_{t-1}}: & \left(\Lambda^{*, *}\left(x_{1,0}^{j}, x_{0,1}^{j}\right), 1 \leq j \leq n-n_{t-1}, \quad \bar{\partial}=0\right), \\
\mathbb{T}^{n_{t-1}-n_{t-2}}: & \left(\Lambda^{*, *}\left(y_{1,0}^{i}, y_{0,1}^{i}\right), n-n_{t-1}+1 \leq i \leq n-n_{t-2}, \quad \bar{\partial}=0\right),
\end{aligned}
$$


where the generators are all of degree 1 and bidegree as indexed. Then, having in mind the previous argumentation for $l=t-1$ and the Hirsch Lemma, we see that a model for $H_{\bar{\partial}}^{*, *}\left(\Gamma_{t-2} \backslash G_{t-2}\right)$ is the differential bigraded algebra

$$
\mathcal{A}^{*, *}=\left(\Lambda^{*, *}\left(x_{1,0}^{j}, x_{0,1}^{j}\right) \otimes \Lambda^{*, *}\left(y_{1,0}^{i}, y_{0,1}^{i}\right), 1 \leq j \leq n-n_{t-1}<i \leq n-n_{t-2}, \bar{\partial}\right),
$$

the differential $\bar{\partial}$ being given as follows:

$$
\left\{\begin{array}{l}
\bar{\partial} x_{1,0}^{j}=\bar{\partial} x_{0,1}^{j}=0, \quad 1 \leq j \leq n-n_{t-1}, \\
\bar{\partial} y_{1,0}^{i}=\sum_{\substack{j, k=1 \\
n-n_{t-1}}} B_{i j k} x_{1,0}^{j} \wedge x_{0,1}^{k}, \quad n-n_{t-1}+1 \leq i \leq n-n_{t-2}, \\
\bar{\partial} y_{0,1}^{i}=\sum_{\substack{j, k=1 \\
j<k}}^{n-n_{t-1}} \bar{A}_{i j k} x_{0,1}^{j} \wedge x_{0,1}^{k}, \quad n-n_{t-1}+1 \leq i \leq n-n_{t-2},
\end{array}\right.
$$

$A_{i j k}$ and $B_{i j k}$ being the structure constants of $\mathfrak{g}$ in (2).

Therefore, the mapping $\mathcal{A}^{*, *} \longrightarrow \Lambda_{\mathbb{C}}^{*, *}\left(\Gamma_{t-2} \backslash G_{t-2}\right)$ given by

$$
\begin{aligned}
& x_{1,0}^{j} \mapsto \omega_{j}, \quad x_{0,1}^{j} \mapsto \bar{\omega}_{j}, \quad 1 \leq j \leq n-n_{t-1}, \\
& y_{1,0}^{i} \mapsto \omega_{i}, \quad y_{0,1}^{i} \mapsto \bar{\omega}_{i}, \quad n-n_{t-1}+1 \leq i \leq n-n_{t-2},
\end{aligned}
$$

defines an isomorphism $H_{\bar{\partial}}^{p, q}\left(\Gamma_{t-2} \backslash G_{t-2}\right) \cong H_{\bar{\partial}}^{p, q}\left(\left(\mathfrak{g} / \mathfrak{a}_{t-2}\right)^{\mathbb{C}}\right)$. If we continue the iteration, we get $H_{\bar{\partial}}^{p, q}\left(\Gamma_{t-3} \backslash G_{t-3}\right) \cong H_{\bar{\partial}}^{p, q}\left(\left(\mathfrak{g} / \mathfrak{a}_{t-3}\right)^{\mathbb{C}}\right)$ at the second step, and finally, at the top of the tower, $H_{\bar{\partial}}^{p, q}(\Gamma \backslash G) \cong H_{\bar{\partial}}^{p, q}\left(\mathfrak{g}^{\mathbb{C}}\right)$, and the proof is complete.

Corollary 21. The differential bigraded algebra $\left(\Lambda^{*, *}\left(\mathfrak{g}^{\mathbb{C}}\right)^{*}, \bar{\partial}\right)$ is a minimal model for the Dolbeault cohomology of $\Gamma \backslash G$.

Proof. It is obvious since $J$ is nilpotent.

Remark 22. This proof can be adapted to the real context if we suppose $\Gamma \backslash G$ to be a compact (real) nilmanifold and take Remark 16 into account. Then, using the classical Hirsch Lemma, Nomizu's theorem will follow: $H^{*}(\Gamma \backslash G, \mathbb{R})$ is canonically isomorphic to $H^{*}(\mathfrak{g})$ (see [CFG5]).

Sakane's theorem [Sa] for the Dolbeault cohomology of a compact complex parallelizable nilmanifold follows easily from our Main Theorem and Remark 17. Suppose that $\Gamma \backslash G$ is a compact complex parallelizable nilmanifold. Then the coefficients $B_{i j k}$ in (2), and hence in (24), are all zero. Therefore, a minimal model for the Dolbeault cohomology $H_{\bar{\partial}}^{*, *}(\Gamma \backslash G)$ is the differential bigraded algebra

$$
\mathcal{A}^{*, *}=\left(\Lambda^{*, *}\left(x_{1,0}^{i}, x_{0,1}^{i}\right), 1 \leq i \leq n, \bar{\partial}\right),
$$

the differential $\bar{\partial}$ being given by

$$
\bar{\partial} x_{1,0}^{i}=0, \quad \bar{\partial} x_{0,1}^{i}=\sum_{j<k<i} \bar{A}_{i j k} x_{0,1}^{j} \wedge x_{0,1}^{k} .
$$

Hence $H_{\bar{\partial}}^{p, q}(\Gamma \backslash G) \cong H^{p, q}\left(\mathcal{A}^{*, *}, \bar{\partial}\right) \cong \Lambda^{p}\left(x_{1,0}^{i}\right) \otimes H^{q}\left(\Lambda\left(x_{0,1}^{i}\right)\right)$, where $\Lambda^{*}\left(x_{1,0}^{i}\right)$ is the exterior algebra generated by $\left\{x_{1,0}^{i} ; 1 \leq i \leq n\right\}$, and $H^{q}\left(\Lambda\left(x_{0,1}^{i}\right)\right)$ is the cohomology of the differential algebra $\left(\Lambda^{*}\left(x_{0,1}^{i}\right), \bar{\partial}\right)$. Thus, 
Theorem 23 ( $\mathrm{Sa}]$ ). Let $\Gamma \backslash G$ be a compact complex parallelizable nilmanifold. Then there is a canonical isomorphism

$$
H_{\bar{\partial}}^{p, q}(\Gamma \backslash G) \cong \Lambda^{p}\left(\mathfrak{g}_{1,0}\right)^{*} \otimes H^{q}\left(\Lambda\left(\mathfrak{g}_{0,1}\right)^{*}\right),
$$

where $H^{q}\left(\Lambda\left(\mathfrak{g}_{0,1}\right)^{*}\right)$ denotes the cohomology of $\left(\mathfrak{g}_{0,1}\right)^{*}$.

\section{EXAMPLES OF COMPACT NILMANIFOLDS WITH NILPOTENT COMPLEX STRUCTURE}

We shall apply all the previous constructions and results to computing the Dolbeault cohomology of some compact nilmanifolds with nilpotent complex structure. Four examples will be developed. The first one is the Iwasawa manifold; it is the simplest non-trivial example of a compact complex parallelizable nilmanifold. The last three examples are compact nilmanifolds with nilpotent complex structure which are not complex parallelizable; in particular, Example 2 is the well known Kodaira-Thurston manifold.

Example 1. The Iwasawa manifold $I_{3}$. Let us consider the structure equations

$$
d \omega_{1}=d \omega_{2}=0, \quad d \omega_{3}=-\omega_{1} \wedge \omega_{2} .
$$

They determine the complex Heisenberg group, that is, the complex nilpotent Lie group $G$ of complex matrices of the form

$$
\left(\begin{array}{ccc}
1 & z_{1} & z_{3} \\
& 1 & z_{2} \\
& & 1
\end{array}\right) .
$$

The Iwasawa manifold is the compact complex parallelizable nilmanifold obtained as $I_{3}=\Gamma \backslash G$, where $\Gamma$ is the subgroup of $G$ consisting of those matrices whose entries are Gaussian integers. In terms of the natural (complex) coordinate functions $z_{1}, z_{2}, z_{3}$ on $G$, we have that the 1 -forms $\omega_{1}, \omega_{2}$ and $\omega_{3}$ can be expressed by

$$
\omega_{1}=d z_{1}, \quad \omega_{2}=d z_{2}, \quad \omega_{3}=d z_{3}-z_{1} d z_{2} .
$$

Since these 1-forms are left invariant on $G$, they descend to the quotient $I_{3}$.

If $\left\{X_{1}, \ldots, \bar{X}_{3}\right\}$ is the basis of left invariant vector fields dual to the basis of 1 -forms $\left\{\omega_{1}, \ldots, \bar{\omega}_{3}\right\}$, then $\mathfrak{a}_{1}=\mathfrak{g}_{1}=\left\{X_{3}, \bar{X}_{3}\right\}$ and $\mathfrak{a}_{2}=\mathfrak{g}_{2}=\mathfrak{g}$; therefore $I_{3}$ is the total space of the holomorphic principal torus bundle

$$
\mathbb{T}^{1} \hookrightarrow I_{3} \stackrel{\pi}{\longrightarrow} \mathbb{T}^{2} .
$$

At the level of Lie groups, the projection $\pi: G \rightarrow \mathbb{C}^{2}$ is given by $\pi\left(z_{1}, z_{2}, z_{3}\right)=$ $\left(z_{1}, z_{2}\right)$, and the right action $G \times \mathbb{C} \rightarrow G$ by $\left(\left(z_{1}, z_{2}, z_{3}\right), w\right) \mapsto\left(z_{1}, z_{2}, z_{3}+w\right)$.

Let $Z$ denote the left invariant complex vector field of type $(1,0)$ on $\mathbb{T}^{1}$; then $\boldsymbol{\omega}=\omega_{3} \otimes Z$ is the canonical (holomorphic) connection on the bundle $I_{3}\left(\mathbb{T}^{2}, \pi, \mathbb{T}^{1}\right)$, and its curvature form is $\boldsymbol{\Omega}=-\left(\omega_{1} \wedge \omega_{2}\right) \otimes Z$. Since $\boldsymbol{\Omega}^{1,1}=0$, the Atiyah class of this holomorphic principal bundle vanishes.

For the Iwasawa manifold $I_{3}$, as total space of a holomorphic principal bundle, the base is $\mathbb{T}^{2}$ and the fibre is $\mathbb{T}^{1}$. Therefore, their Dolbeault cohomology groups are

$$
\begin{aligned}
& H_{\bar{\partial}}^{*, *}(B)=H_{\bar{\partial}}^{*, *}\left(\mathbb{T}^{2}\right) \cong H\left(\Lambda^{*, *}\left(x_{1,0}^{1}, x_{1,0}^{2}, x_{0,1}^{1}, x_{0,1}^{2}\right), \bar{\partial}=0\right), \\
& H_{\bar{\partial}}^{*, *}(F)=H_{\bar{\partial}}^{*, *}\left(\mathbb{T}^{1}\right) \cong H\left(\Lambda^{*, *}\left(y_{1,0}, y_{0,1}\right), \bar{\partial}=0\right),
\end{aligned}
$$


where all the generators have total degree 1 and bidegree as indexed; hence, a model for the Dolbeault cohomology of $I_{3}$ is the differential bigraded algebra

$$
\left(\Lambda^{*, *}\left(x_{1,0}^{1}, x_{1,0}^{2}, x_{0,1}^{1}, x_{0,1}^{2}\right) \otimes \Lambda^{* * *}\left(y_{1,0}, y_{0,1}\right), \bar{\partial}\right),
$$

with $\bar{\partial}$ given by

$$
\begin{aligned}
\bar{\partial} x_{1,0}^{1} & =\bar{\partial} x_{1,0}^{2}=\bar{\partial} x_{0,1}^{1}=\bar{\partial} x_{0,1}^{2}=\bar{\partial} y_{1,0}=0, \\
\bar{\partial} y_{0,1} & =-x_{0,1}^{1} \wedge x_{0,1}^{2} .
\end{aligned}
$$

This is indeed a minimal model for the Dolbeault cohomology of $I_{3}$.

A straightforward computation allows us to find explicitly each $H_{\bar{\partial}}^{p, q}\left(I_{3}\right)$ (see Co for the details); the dimensions of these groups (see also [FG], $[\mathrm{Sa}]$ ) are

$$
\begin{aligned}
& h^{3,3}\left(I_{3}\right)=1, h^{3,2}\left(I_{3}\right)=h^{0,1}\left(I_{3}\right)=2, h^{2,3}\left(I_{3}\right)=h^{1,0}\left(I_{3}\right)=3, \\
& h^{3,1}\left(I_{3}\right)=h^{0,2}\left(I_{3}\right)=2, h^{1,3}\left(I_{3}\right)=h^{2,0}\left(I_{3}\right)=3, \\
& h^{3,0}\left(I_{3}\right)=h^{0,3}\left(I_{3}\right)=1, h^{2,1}\left(I_{3}\right)=h^{1,2}\left(I_{3}\right)=6, \\
& h^{2,2}\left(I_{3}\right)=h^{1,1}\left(I_{3}\right)=6 .
\end{aligned}
$$

In view of this list, we note that complex conjugation does not define in general an isomorphism between $H_{\bar{\partial}}^{p, q}\left(I_{3}\right)$ and $H_{\bar{\partial}}^{q, p}\left(I_{3}\right)$.

Moreover, it is easy to check that the minimal model for the Dolbeault cohomology of $I_{3}$ is in fact not formal. For example, the cohomology class of $x_{0,1}^{1} \wedge y_{0,1}$ defines the nonzero Massey product $\left\langle x_{0,1}^{1}, x_{0,1}^{1}, x_{0,1}^{2}\right\rangle$.

More examples of compact complex parallelizable nilmanifolds can be constructed in the same way. For a complete list of these manifolds up to complex dimension 5 , see [Na].

Example 2. The Kodaira-Thurston manifold $K$. The simplest example of a compact nilmanifold with nilpotent complex structures which is real parallelizable but not complex parallelizable is the following. Let us consider the structure equations

$$
d \omega_{1}=0, \quad d \omega_{2}=\omega_{1} \wedge \bar{\omega}_{1} .
$$

They determine the simply-connected connected nilpotent Lie group $G$ of complex matrices of the form

$$
\left(\begin{array}{ccc}
1 & \bar{z}_{1} & z_{2} \\
& 1 & z_{1} \\
& & 1
\end{array}\right) .
$$

We note that this Lie group $G$ is not a complex Lie group, since right translations are not holomorphic. The Kodaira-Thurston manifold $K$ is the compact nilmanifold with nilpotent complex structure obtained as $K=\Gamma \backslash G$, where $\Gamma$ is the subgroup of $G$ consisting of those matrices whose entries are Gaussian integers. (This manifold was the first known example of a compact symplectic manifold which is also a complex manifold with no positive definite Kähler metric [Ko, Th, CFG1].) The functions $z_{1}, z_{2}$ are natural complex coordinates on $G$. In terms of $d z_{1}$ and $d z_{2}$, the 1 -forms $\omega_{1}$ and $\omega_{2}$ can be expressed by

$$
\omega_{1}=d z_{1}, \quad \omega_{2}=d z_{2}-\bar{z}_{1} d z_{1} .
$$

Moreover, these 1-forms descend to the quotient $K$ because they are left invariant on $G$. 
If $\left\{X_{1}, \bar{X}_{1}, X_{2}, \bar{X}_{2}\right\}$ is the basis of left invariant vector fields dual to the basis of 1 -forms $\left\{\omega_{1}, \bar{\omega}_{1}, \omega_{2}, \bar{\omega}_{2}\right\}$, then $\mathfrak{a}_{1}=\mathfrak{g}_{1}=\left\{X_{2}, \bar{X}_{2}\right\}, \mathfrak{a}_{2}=\mathfrak{g}_{2}=\mathfrak{g}$. Therefore $K$ is the total space of the holomorphic principal bundle

$$
\mathbb{T}^{1} \hookrightarrow K \stackrel{\pi}{\longrightarrow} \mathbb{T}^{1} .
$$

At the level of Lie groups, the projection $\pi: G \rightarrow \mathbb{C}$ is given by $\pi\left(z_{1}, z_{2}\right)=z_{1}$, and the right action $G \times \mathbb{C} \rightarrow G$ by $\left(\left(z_{1}, z_{2}\right), w\right) \mapsto\left(z_{1}, z_{2}+w\right)$.

Let $Z$ denote the left invariant complex vector field of type $(1,0)$ on $\mathbb{T}^{1}$; then, $\boldsymbol{\omega}=\omega_{2} \otimes Z$ is the canonical (non-holomorphic) connection on the holomorphic principal bundle $K\left(\mathbb{T}^{1}, \pi, \mathbb{T}^{1}\right)$, and its curvature form is given by $\boldsymbol{\Omega}=\left(\omega_{1} \wedge \bar{\omega}_{1}\right) \otimes Z$. Since $\left[\omega_{1} \wedge \bar{\omega}_{1}\right] \in H_{\bar{\partial}}^{1,1}\left(\mathbb{T}^{1}, \mathbb{C}\right.$ ) is nonzero (here $\mathbb{C}$ is identified to the Lie algebra of $\mathbb{T}^{1}$ ), this holomorphic principal bundle has nonvanishing Atiyah class and hence does not admit holomorphic connections.

For the Kodaira-Thurston manifold $K$, as total space of a holomorphic principal bundle, the base and the fibre both are $\mathbb{T}^{1}$. Therefore, their Dolbeault cohomology groups are

$$
\begin{aligned}
& H_{\bar{\partial}}^{*, *}(B)=H_{\bar{\partial}}^{*, *}\left(\mathbb{T}^{1}\right) \cong H\left(\Lambda^{*, *}\left(x_{1,0}, x_{0,1}\right), \bar{\partial}=0\right), \\
& H_{\bar{\partial}}^{*, *}(F)=H_{\bar{\partial}}^{*, *}\left(\mathbb{T}^{1}\right) \cong H\left(\Lambda^{*, *}\left(y_{1,0}, y_{0,1}\right), \bar{\partial}=0\right),
\end{aligned}
$$

where the generators have total degree 1 and bidegree as indexed; hence, a minimal model for the Dolbeault cohomology of $K$ is the differential bigraded algebra $\left(\Lambda^{*, *}\left(x_{1,0}, x_{0,1}\right) \otimes \Lambda^{*, *}\left(y_{1,0}, y_{0,1}\right), \bar{\partial}\right)$, with $\bar{\partial}$ given by

$$
\bar{\partial} x_{1,0}=\bar{\partial} x_{0,1}=\bar{\partial} y_{0,1}=0, \quad \bar{\partial} y_{1,0}=x_{1,0} \wedge x_{0,1} .
$$

The explicit list of the groups $H_{\bar{\partial}}^{p, q}(K)$ is given in [C]. Their dimensions are:

$$
\begin{aligned}
& h^{2,2}(K)=1, h^{2,1}(K)=h^{0,1}(K)=2, h^{1,2}(K)=h^{1,0}(K)=1, \\
& h^{2,0}(K)=h^{0,2}(K)=1, h^{1,1}(K)=2 .
\end{aligned}
$$

Again we remark that complex conjugation does not define in general an isomorphism between $H_{\bar{\partial}}^{p, q}(K)$ and $H_{\bar{\partial}}^{q, p}(K)$. Also, the minimal model is not formal. For example, the cohomology class of $x_{1,0} \wedge y_{1,0}$ defines the nonzero Massey product $\left\langle x_{1,0}, x_{1,0}, x_{0,1}\right\rangle$.

Example 3. Let us consider the structure equations

$$
\begin{aligned}
& d \omega_{1}=0, \\
& d \omega_{2}=\omega_{1} \wedge \bar{\omega}_{1}, \\
& d \omega_{3}=\omega_{1} \wedge \omega_{2}+\omega_{1} \wedge \bar{\omega}_{2} ;
\end{aligned}
$$

they determine the (real) nilpotent Lie group $G$ of complex matrices of the form

$$
\left(\begin{array}{cccccc}
1 & \bar{z}_{1} & z_{2} & z_{1} & \frac{1}{2} z_{1}^{2} & z_{3} \\
& 1 & z_{1} & 0 & 0 & \frac{1}{2} z_{1}^{2} \\
& & 1 & 0 & 0 & z_{1} \\
& & & 1 & z_{1} & -\bar{z}_{2} \\
& & & & 1 & -\bar{z}_{1} \\
& & & & & 1
\end{array}\right) .
$$

Then $N^{3}=\Gamma \backslash G$, where $\Gamma$ denotes the subgroup of $G$ consisting of those matrices with Gaussian integers as entries, is a compact nilmanifold with a nilpotent complex structure of complex dimension 3 . 
If $\left\{X_{1}, \ldots, \bar{X}_{3}\right\}$ is the basis of left invariant vector fields dual to the basis of 1 -forms $\left\{\omega_{1}, \ldots, \bar{\omega}_{3}\right\}$, then

$$
\begin{aligned}
& \mathfrak{a}_{1}=\left\{X_{3}, \bar{X}_{3}\right\} \subset \mathfrak{g}_{1}=\left\{X_{2}-\bar{X}_{2}, X_{3}, \bar{X}_{3}\right\}, \\
& \mathfrak{a}_{2}=\left\{X_{2}, \bar{X}_{2}, X_{3}, \bar{X}_{3}\right\} \subset \mathfrak{g}_{2}=\mathfrak{g}, \\
& \mathfrak{a}_{3}=\mathfrak{g} .
\end{aligned}
$$

Therefore, we obtain in this case the following tower of holomorphic principal bundles:

$$
\begin{array}{r}
\mathbb{T}^{1} \hookrightarrow N^{3} \\
\downarrow \\
\mathbb{T}^{1} \hookrightarrow N^{2} \\
\downarrow \\
\mathbb{T}^{1}
\end{array}
$$

Here $N^{2}=\Gamma_{1} \backslash G_{1}$, where $G_{1}$ is the nilpotent Lie group defined by the structure equations $d \omega_{1}=0, d \omega_{2}=\omega_{1} \wedge \bar{\omega}_{1}$; that is, $N^{2}$ is the Kodaira-Thurston manifold $K$ considered in Example 2.

At the level of Lie groups, the projection maps in this tower are given as follows:

$$
\begin{aligned}
& \pi_{1}: G \longrightarrow G_{1}: \quad\left(z_{1}, z_{2}, z_{3}\right) \mapsto\left(z_{1}, z_{2}\right), \\
& \pi_{2}: G_{1} \longrightarrow \mathbb{C}: \quad\left(z_{1}, z_{2}\right) \mapsto z_{1},
\end{aligned}
$$

and the right actions by

$$
\begin{aligned}
G \times \mathbb{C} \longrightarrow G: & \left(\left(z_{1}, z_{2}, z_{3}\right), w_{3}\right) \mapsto\left(z_{1}, z_{2}, z_{3}+w_{3}\right), \\
G_{1} \times \mathbb{C} \longrightarrow G_{1}: & \left(\left(z_{1}, z_{2}\right), w_{2}\right) \mapsto\left(z_{1}, z_{2}+w_{2}\right) .
\end{aligned}
$$

The canonical (non-holomorphic) connections on this tower are given as follows. The connection form on $K\left(\mathbb{T}^{1}, \pi_{2}, \mathbb{T}^{1}\right)$ is $\omega=\omega_{2} \otimes Z_{2}$, and its curvature form is $\boldsymbol{\Omega}=\left(\omega_{1} \wedge \bar{\omega}_{1}\right) \otimes Z_{2}$. On the bundle $N^{3}\left(K, \pi_{1}, \mathbb{T}^{1}\right)$ the connection form is $\boldsymbol{\omega}=\omega_{3} \otimes Z_{3}$, and its curvature form is $\boldsymbol{\Omega}=\left(\omega_{1} \wedge \omega_{2}+\omega_{1} \wedge \bar{\omega}_{2}\right) \otimes Z_{3}$. Again, each bundle at this tower has nonvanishing Atiyah class, and hence they cannot admit holomorphic connections.

In order to compute $H_{\bar{\partial}}^{*, *}\left(N^{3}\right)$ we proceed by iteration from the bottom to the top of the tower. A minimal model for the Dolbeault cohomology of $K$ was constructed in Example 2. Now, at the second step, the bundle has base $K$ and fibre $\mathbb{T}^{1}$. Hence, a minimal model for the Dolbeault cohomology of $N^{3}$ is

$$
\left(\Lambda^{*, *}\left(x_{1,0}, x_{0,1}\right) \otimes \Lambda^{*, *}\left(y_{1,0}, y_{0,1}\right) \otimes \Lambda^{*, *}\left(z_{1,0}, z_{0,1}\right), \bar{\partial}\right),
$$

with generators of total degree 1 and bidegree as indexed, and $\bar{\partial}$ given by

$$
\begin{aligned}
\bar{\partial} x_{1,0} & =\bar{\partial} x_{0,1}=\bar{\partial} y_{0,1}=0, \\
\bar{\partial} y_{1,0} & =x_{1,0} \wedge x_{0,1}, \\
\bar{\partial} z_{1,0} & =x_{1,0} \wedge y_{0,1}, \\
\bar{\partial} z_{0,1} & =x_{0,1} \wedge y_{0,1} .
\end{aligned}
$$

This minimal model is again not formal. In fact, the cohomology class of $x_{1,0} \wedge$ $z_{1,0}$ defines the nonzero Massey product $\left\langle x_{1,0}, x_{1,0}, y_{0,1}\right\rangle$. 
Example 4. Let $G$ be a simply-connected connected nilpotent Lie group determined by the structure equations

$$
\begin{aligned}
& d \mu_{1}=d \mu_{2}=0 \\
& d \mu_{3}=\mu_{1} \wedge \bar{\mu}_{1}, \\
& d \mu_{4}=\mu_{1} \wedge\left(\mu_{2}+\bar{\mu}_{2}\right)+\mu_{1} \wedge\left(\mu_{3}+\bar{\mu}_{3}\right), \\
& d \mu_{5}=\mu_{1} \wedge\left(-\mu_{2}+\bar{\mu}_{2}\right)+\mu_{1} \wedge \mu_{4} .
\end{aligned}
$$

Let $\Gamma$ be a lattice in $G$, and $N^{5}=\Gamma \backslash G$. Then $N^{5}$ is a compact nilmanifold with a nilpotent complex structure of complex dimension 5 .

If $\left\{Z_{1}, \ldots, \bar{Z}_{5}\right\}$ is the basis of left invariant vector fields dual to the basis of 1 -forms $\left\{\mu_{1}, \ldots, \bar{\mu}_{5}\right\}$, then

$$
\begin{aligned}
\mathfrak{a}_{1} & =\left\{Z_{5}, \bar{Z}_{5}\right\} \subset \mathfrak{g}_{1}=\left\{Z_{2}+\bar{Z}_{2}-2 Z_{3}, Z_{2}-Z_{3}+Z_{4}-\bar{Z}_{4}, Z_{3}-\bar{Z}_{3}, Z_{5}, \bar{Z}_{5}\right\}, \\
\mathfrak{a}_{2} & =\left\{Z_{2}-Z_{3}, \bar{Z}_{2}-\bar{Z}_{3}, Z_{4}, \bar{Z}_{4}, Z_{5}, \bar{Z}_{5}\right\} \subset \mathfrak{g}_{2} \\
& =\left\{Z_{2}-\bar{Z}_{2}, Z_{2}+\bar{Z}_{2}-2 Z_{3}, Z_{3}-\bar{Z}_{3}, Z_{4}, \bar{Z}_{4}, Z_{5}, \bar{Z}_{5}\right\} \\
& =\left\{Z_{2}-Z_{3}, \bar{Z}_{2}-\bar{Z}_{3}, Z_{3}-\bar{Z}_{3}, Z_{4}, \bar{Z}_{4}, Z_{5}, \bar{Z}_{5}\right\}, \\
\mathfrak{a}_{3} & =\left\{Z_{2}, \bar{Z}_{2}, Z_{3}, \bar{Z}_{3}, Z_{4}, \bar{Z}_{4}, Z_{5}, \bar{Z}_{5}\right\} \subset \mathfrak{g}_{3}=\mathfrak{g}, \\
\mathfrak{a}_{4} & =\mathfrak{g} .
\end{aligned}
$$

Now, we shall consider a new basis $\left\{X_{1}, \ldots, \bar{X}_{5}\right\}$ for $\mathfrak{g}$ such that $\left\{X_{5}, \bar{X}_{5}\right\}$ is a basis for $\mathfrak{a}_{1},\left\{X_{3}, \bar{X}_{3}, X_{4}, \bar{X}_{4}, X_{5}, \bar{X}_{5}\right\}$ is a basis for $\mathfrak{a}_{2}$ and $\left\{X_{2}, \bar{X}_{2}, X_{3}, \bar{X}_{3}, X_{4}, \bar{X}_{4}, X_{5}\right.$, $\left.\bar{X}_{5}\right\}$ is a basis for $\mathfrak{a}_{3}$. For that, we define the 1-forms $\omega_{i}, 1 \leq i \leq 5$, as follows:

$$
\omega_{1}=\mu_{1}, \quad \omega_{2}=\mu_{2}+\mu_{3}, \quad \omega_{3}=\mu_{2}-\mu_{3}, \quad \omega_{4}=\mu_{4}, \quad \omega_{5}=\mu_{5} .
$$

Then $\left\{\omega_{5}, \bar{\omega}_{5}\right\}$ is a basis for $\mathfrak{a}_{1}^{*},\left\{\omega_{3}, \bar{\omega}_{3}, \omega_{4}, \bar{\omega}_{4}, \omega_{5}, \bar{\omega}_{5}\right\}$ is a basis for $\mathfrak{a}_{2}^{*},\left\{\omega_{2}, \bar{\omega}_{2}, \omega_{3}, \bar{\omega}_{3}\right.$, $\left.\omega_{4}, \bar{\omega}_{4}, \omega_{5}, \bar{\omega}_{5}\right\}$ is a basis for $\mathfrak{a}_{3}^{*}$, and $\left\{\omega_{i}, \bar{\omega}_{i} ; 1 \leq i \leq 5\right\}$ is a basis for the 1 -forms on $\mathfrak{g}\left(=\mathfrak{a}_{4}\right)$ satisfying

$$
\begin{aligned}
& d \omega_{1}=0 \\
& d \omega_{2}=\omega_{1} \wedge \bar{\omega}_{1}, \\
& d \omega_{3}=-\omega_{1} \wedge \bar{\omega}_{1}, \\
& d \omega_{4}=\omega_{1} \wedge\left(\omega_{2}+\bar{\omega}_{2}\right), \\
& d \omega_{5}=\frac{1}{2} \omega_{1} \wedge\left(-\omega_{2}-\omega_{3}+2 \omega_{4}+\bar{\omega}_{2}+\bar{\omega}_{3}\right) .
\end{aligned}
$$

Let $\left\{X_{1}, \ldots, \bar{X}_{5}\right\}$ be the basis for $\mathfrak{g}$ dual to the basis $\left\{\omega_{1}, \ldots, \bar{\omega}_{5}\right\}$. Then, $\left\{X_{1}, \ldots\right.$, $\left.\bar{X}_{5}\right\}$ is the desired basis for $\mathfrak{g}$.

Therefore, we have the following tower of holomorphic principal bundles:

$$
\begin{array}{r}
\mathbb{T}^{1} \hookrightarrow N^{5} \\
\downarrow \\
\mathbb{T}^{2} \hookrightarrow N^{4} \\
\downarrow \\
\mathbb{T}^{1} \hookrightarrow N^{2} \\
\downarrow \\
\mathbb{T}^{1}
\end{array}
$$


Here $N^{4}=\Gamma_{1} \backslash G_{1}$ and $N^{2}=\Gamma_{2} \backslash G_{2}$, where $G_{1}$ and $G_{2}$ are the nilpotent Lie groups defined by the structure equations

$$
d \omega_{1}=0, \quad d \omega_{2}=\omega_{1} \wedge \bar{\omega}_{1}, \quad d \omega_{3}=-\omega_{1} \wedge \bar{\omega}_{1}, \quad d \omega_{4}=\omega_{1} \wedge\left(\omega_{2}+\bar{\omega}_{2}\right),
$$

and

$$
d \omega_{1}=0, \quad d \omega_{2}=\omega_{1} \wedge \bar{\omega}_{1},
$$

respectively. That is, $N^{2}$ is the Kodaira-Thurston manifold $K$.

The canonical (non-holomorphic) connections on the tower are given as follows. The connection form on $K\left(\mathbb{T}^{1}, \pi_{3}, \mathbb{T}^{1}\right)$ is $\boldsymbol{\omega}=\omega_{2} \otimes W_{2}$, and its curvature form is

$$
\boldsymbol{\Omega}=\left(\omega_{1} \wedge \bar{\omega}_{1}\right) \otimes W_{2} .
$$

On the bundle $N^{4}\left(K, \pi_{2}, \mathbb{T}^{2}\right)$ the connection form is $\boldsymbol{\omega}=\omega_{3} \otimes W_{3}+\omega_{4} \otimes W_{4}$, and its curvature form is

$$
\boldsymbol{\Omega}=-\left(\omega_{1} \wedge \bar{\omega}_{1}\right) \otimes W_{3}+\left(\omega_{1} \wedge \omega_{2}+\omega_{1} \wedge \bar{\omega}_{2}\right) \otimes W_{4} .
$$

On the bundle $N^{5}\left(N^{4}, \pi_{1}, \mathbb{T}^{1}\right)$ the connection form is $\boldsymbol{\omega}=\omega_{5} \otimes W_{5}$, and its curvature form is

$$
\boldsymbol{\Omega}=\frac{1}{2}\left(-\omega_{1} \wedge \omega_{2}-\omega_{1} \wedge \omega_{3}+2 \omega_{1} \wedge \omega_{4}+\omega_{1} \wedge \bar{\omega}_{2}+\omega_{1} \wedge \bar{\omega}_{3}\right) \otimes W_{5}
$$

Again, each bundle at this tower has nonvanishing Atiyah class, and hence they cannot admit holomorphic connections.

In order to compute $H_{\bar{\partial}}^{*, *}\left(N^{5}\right)$ we proceed by iteration from the bottom to the top of the tower. A minimal model for the Dolbeault cohomology of $K$ was constructed in Example 2. At the second step, the bundle has base $K$ and fibre $\mathbb{T}^{2}$. Hence, a minimal model for the Dolbeault cohomology of $N^{4}$ is

$$
\left(\Lambda^{* * *}\left(x_{1,0}, x_{0,1}\right) \otimes \Lambda^{*, *}\left(y_{1,0}, y_{0,1}\right) \otimes \Lambda^{*, *}\left(z_{1,0}^{1}, z_{1,0}^{2}, z_{0,1}^{1}, z_{0,1}^{2}\right), \bar{\partial}\right),
$$

with generators of total degree 1 and bidegree as indexed, and $\bar{\partial}$ given by

$$
\begin{aligned}
\bar{\partial} x_{1,0} & =\bar{\partial} x_{0,1}=\bar{\partial} y_{0,1}=\bar{\partial} z_{0,1}^{1}=0, \\
\bar{\partial} y_{1,0} & =x_{1,0} \wedge x_{0,1}, \\
\bar{\partial} z_{1,0}^{1} & =-x_{1,0} \wedge x_{0,1}, \\
\bar{\partial} z_{1,0}^{2} & =x_{1,0} \wedge y_{0,1}, \\
\bar{\partial} z_{0,1}^{2} & =x_{0,1} \wedge y_{0,1} .
\end{aligned}
$$

At the third step, the bundle has base $N^{4}$ and fibre $\mathbb{T}^{1}$. Hence, a minimal model for the Dolbeault cohomology of $N^{5}$ is

$$
\left(\Lambda^{*, *}\left(x_{1,0}, x_{0,1}\right) \otimes \Lambda^{*, *}\left(y_{1,0}, y_{0,1}\right) \otimes \Lambda^{*, *}\left(z_{1,0}^{1}, z_{1,0}^{2}, z_{0,1}^{1}, z_{0,1}^{2}\right) \otimes \Lambda^{*, *}\left(t_{1,0}, t_{0,1}\right), \bar{\partial}\right),
$$

where the generators $t_{1,0}, t_{0,1}$ have total degree 1 and bidegree as indexed, and $\bar{\partial}$ is given by

$$
\begin{aligned}
& \bar{\partial} t_{1,0}=\frac{1}{2} x_{1,0} \wedge y_{0,1}+\frac{1}{2} x_{1,0} \wedge z_{0,1}^{1} \\
& \bar{\partial} t_{0,1}=-\frac{1}{2} x_{0,1} \wedge y_{0,1}-\frac{1}{2} x_{0,1} \wedge z_{0,1}^{1}+x_{0,1} \wedge z_{0,1}^{2} .
\end{aligned}
$$

This minimal model is again not formal. In fact, the cohomology class of $x_{1,0} \wedge$ $z_{1,0}^{2}$ defines the nonzero Massey product $\left\langle x_{1,0}, x_{1,0}, y_{0,1}\right\rangle$. 


\section{EXAMPLES OF COMPACT COMPLEX NILMANIFOLDS WITH NO NILPOTENT COMPLEX STRUCTURE}

In this section we exhibit two examples of compact complex nilmanifolds, $L^{6}$ and $M^{10}$, whose complex structures are not nilpotent. Therefore, the Main Theorem of this paper cannot be applied to compute the Dolbeault cohomology groups of $L^{6}$ and $M^{10}$. Moreover, we prove that the compact nilmanifold $L^{6}$ admits no nilpotent complex structures.

The compact complex nilmanifold $L^{6}$. Let $G$ be the 6 -dimensional simplyconnected connected 3-step nilpotent Lie group defined by the structure equations

$$
\left\{\begin{array}{l}
d \alpha_{1}=d \alpha_{2}=d \alpha_{3}=0 \\
d \alpha_{4}=\alpha_{1} \wedge \alpha_{3} \\
d \alpha_{5}=\alpha_{2} \wedge \alpha_{3} \\
d \alpha_{6}=\alpha_{1} \wedge \alpha_{4}+\alpha_{2} \wedge \alpha_{5}
\end{array}\right.
$$

Let $\left\{X_{i} ; 1 \leq i \leq 6\right\}$ be the basis of left invariant vector fields on $G$ dual to the basis $\left\{\alpha_{i} ; 1 \leq i \leq 6\right\}$. Then, $\mathfrak{g}_{1}=\left\{X_{6}\right\}$, and thus $\operatorname{dim} \mathfrak{g}_{1}=1$.

Next, we consider the left invariant integrable almost complex structure $J$ on $G$ defined by

$$
J X_{1}=X_{2}, \quad J X_{4}=X_{5}, \quad J X_{3}=X_{6} .
$$

A complex basis $\left\{\omega_{i} ; 1 \leq i \leq 3\right\}$ for the complex forms on $\mathfrak{g}^{\mathbb{C}}, \mathfrak{g}=$ Lie algebra of $G$, is given by

$$
\left\{\begin{array}{l}
\omega_{1}=\alpha_{1}+\sqrt{-1} \alpha_{2}, \\
\omega_{2}=\alpha_{4}+\sqrt{-1} \alpha_{5}, \\
\omega_{3}=\alpha_{3}+\sqrt{-1} \alpha_{6} .
\end{array}\right.
$$

In terms of these forms, the structure equations of $G$ are

$$
\left\{\begin{array}{l}
d \omega_{1}=0 \\
d \omega_{2}=\frac{1}{2}\left(\omega_{1} \wedge \omega_{3}+\omega_{1} \wedge \bar{\omega}_{3}\right), \\
d \omega_{3}=\frac{\sqrt{-1}}{2}\left(\omega_{1} \wedge \bar{\omega}_{2}+\bar{\omega}_{1} \wedge \omega_{2}\right),
\end{array}\right.
$$

and they allow us, by integration, to describe $G$ as the (real) nilpotent Lie group of complex matrices of the form

$$
\left(\begin{array}{ccccc}
1 & -\frac{\sqrt{-1}}{2} z_{1} & -\frac{\sqrt{-1}}{2} \bar{z}_{1} & -\frac{\sqrt{-1}}{4} z_{1} \bar{z}_{1} & z_{3} \\
1 & 0 & -\frac{1}{2} \bar{z}_{1} & \bar{z}_{2} \\
& 1 & -\frac{1}{2} z_{1} & z_{2} \\
& & 1 & z_{3}+\bar{z}_{3} \\
& & & 1
\end{array}\right)
$$

The functions $z_{1}, z_{2}, z_{3}$ are the complex coordinates on $G$ which correspond to the integrable almost complex structure $J$, and the 1 -forms $\omega_{1}, \omega_{2}, \omega_{3}$ can be expressed 
by

$$
\left\{\begin{array}{l}
\omega_{1}=d z_{1} \\
\omega_{2}=d z_{2}+\frac{1}{2} z_{1}\left(d z_{3}+d \bar{z}_{3}\right), \\
\omega_{3}=d z_{3}+\frac{\sqrt{-1}}{2}\left(z_{1} d \bar{z}_{2}+\bar{z}_{1} d z_{2}+\frac{1}{2} z_{1} \bar{z}_{1}\left(d z_{3}+d \bar{z}_{3}\right)\right) .
\end{array}\right.
$$

Since the structure equations (25) are not of the form (2), it follows that the integrable almost complex structure $J$ on $G$ is not nilpotent. Furthermore, it is easy to check that the first term $\mathfrak{a}_{1}$ in the series $\left\{\mathfrak{a}_{l} ; l \geq 0\right\}$ is $\mathfrak{a}_{1}=0\left(=\mathfrak{a}_{l}\right.$ for $\left.l \geq 0\right)$ in this case, and hence, taking into account Lemma 9 and Theorem 12, the complex structure $J$ on $G$ cannot be nilpotent. In fact, since $\operatorname{dim} \mathfrak{g}_{1}=1$, Proposition 10 and Theorem 12 imply that $G$ does not admit nilpotent (left invariant) complex structures.

Looking at (26), it is easy to see that there exists a lattice $\Gamma \subset G$ of maximal rank, and therefore $L^{6}=\Gamma \backslash G$ is a compact complex nilmanifold with no nilpotent complex structures.

Nevertheless, the compact complex manifold $L^{6}$ can still be described as the total space of a (non-holomorphic) principal bundle $\mathbb{T}^{2} \hookrightarrow L^{6} \stackrel{\pi}{\longrightarrow} \mathbb{T}$, as follows.

At the level of Lie groups, the fibration is defined as $\mathbb{C}^{2} \hookrightarrow G \stackrel{\pi}{\longrightarrow} \mathbb{C}$, where the projection map $\pi: G \rightarrow \mathbb{C}$ is given by $\pi\left(z_{1}, z_{2}, z_{3}\right)=z_{1}$, and the right action $G \times \mathbb{C}^{2} \rightarrow G$ of $\mathbb{C}^{2}$ on $G$ by

$$
\begin{aligned}
\left(\left(z_{1}, z_{2}, z_{3}\right),\left(w_{1}, w_{2}\right)\right) \mapsto & \left(z_{1}, z_{2}+w_{1}-\frac{1}{2} z_{1}\left(w_{2}+\bar{w}_{2}\right),\right. \\
& \left.z_{3}+w_{2}+\frac{\sqrt{-1}}{2}\left(\frac{1}{2} z_{1} \bar{z}_{1}\left(w_{2}+\bar{w}_{2}\right)-z_{1} \bar{w}_{1}-\bar{z}_{1} w_{1}\right)\right),
\end{aligned}
$$

which is not holomorphic. Hence the fibration, already at the level of Lie groups, is not holomorphic either, and the Main Theorem cannot be applied.

Remark 24. It must be remarked that $\operatorname{dim} \mathfrak{a}_{1}=0$ is not the relevant fact to prevent the existence of some nilpotent (left invariant) complex structure on $G$. The relevant fact is that $\operatorname{dim} \mathfrak{g}_{1}=1$, which conflicts with Proposition 10 and Theorem 12 .

The compact complex nilmanifold $M^{10}$. Let us consider the 10-dimensional simply-connected connected nilpotent Lie group $G$ defined by the structure equations

$$
\left\{\begin{array}{l}
d \alpha_{1}=d \alpha_{2}=d \alpha_{3}=d \beta_{1}=0, \\
d \alpha_{4}=\alpha_{1} \wedge \alpha_{3}, \\
d \alpha_{5}=\alpha_{2} \wedge \alpha_{3}, \\
d \alpha_{6}=\alpha_{1} \wedge \alpha_{4}+\alpha_{2} \wedge \alpha_{5}, \\
d \beta_{2}=\alpha_{1} \wedge \beta_{1} \\
d \beta_{3}=\alpha_{2} \wedge \beta_{1} \\
d \beta_{4}=\alpha_{1} \wedge \beta_{2}+\alpha_{2} \wedge \beta_{3} .
\end{array}\right.
$$

Let $\left\{X_{i}, Y_{j} ; 1 \leq i \leq 6,1 \leq j \leq 4\right\}$ be the basis of left invariant vector fields on $G$ dual to the basis $\left\{\alpha_{i}, \beta_{j} ; 1 \leq i \leq 6,1 \leq j \leq 4\right\}$. Then, $\mathfrak{g}_{1}=\left\{X_{6}, Y_{4}\right\}$, $\mathfrak{g}_{2}=\left\{X_{4}, X_{5}, X_{6}, Y_{2}, Y_{3}, Y_{4}\right\}$ and $\mathfrak{g}_{3}=\mathfrak{g}$. Therefore, $\operatorname{dim} \mathfrak{g}_{1}=2, \operatorname{dim} \mathfrak{g}_{2}=6$ 
and $\operatorname{dim} \mathfrak{g}_{3}=10$. Let us now define the left invariant integrable almost complex structures $J$ and $\hat{J}$ on $G$ by

$$
J X_{1}=X_{2}, \quad J X_{4}=X_{5}, \quad J X_{3}=X_{6}, \quad J Y_{1}=Y_{4}, \quad J Y_{2}=Y_{3},
$$

and

$$
\hat{J} X_{1}=X_{2}, \quad \hat{J} X_{3}=Y_{1}, \quad \hat{J} X_{4}=X_{5}, \quad \hat{J} X_{6}=Y_{4}, \quad \hat{J} Y_{2}=Y_{3},
$$

respectively. Then, one easily checks that the series $\left\{\mathfrak{a}_{l}(J) ; l \geq 0\right\}$ of $J$ satisfies $\mathfrak{a}_{l}(J)=0$ for $l \geq 0$, and hence the complex structure $J$ on $G$ is not nilpotent. However, the series $\left\{\mathfrak{a}_{l}(\hat{J}) ; l \geq 0\right\}$ associated to $\hat{J}$ satisfies $\mathfrak{a}_{l}(\hat{J})=\mathfrak{g}_{l}$ for $l \geq 0$, and thus $\mathfrak{a}_{3}(\hat{J})=\mathfrak{g}$ and $\hat{J}$ is nilpotent.

From equations (27) and Mal'cev's theorem $\mathrm{Ma}$ it follows that there is a discrete subgroup $\Gamma$ of $G$ such that the quotient space $\Gamma \backslash G$ is compact. We define $M^{10}$ (respectively $N^{10}$ ) to be the compact complex nilmanifold $\Gamma \backslash G$ with the complex structure defined from $J$ (respectively $\hat{J}$ ) on $G$ by passing to the quotient. Then, the complex structure on $M^{10}$ is not nilpotent; however, the complex structure on $N^{10}$ is nilpotent.

Moreover, denote by $\eta_{i}(1 \leq i \leq 5)$ the left invariant 1 -forms on $G$ of type $(1,0)$ with respect to $\hat{J}$, defined by

$$
\left\{\begin{array}{l}
\eta_{1}=\alpha_{1}+\sqrt{-1} \alpha_{2} \\
\eta_{2}=\alpha_{3}+\sqrt{-1} \beta_{1}, \\
\eta_{3}=\alpha_{4}+\sqrt{-1} \alpha_{5} \\
\eta_{4}=\beta_{2}+\sqrt{-1} \beta_{3} \\
\eta_{5}=\alpha_{6}+\sqrt{-1} \beta_{4} .
\end{array}\right.
$$

Then, in terms of the 1 -forms $\eta_{i}$, the compact complex nilmanifold $N^{10}$ can be defined by the equations

$$
\left\{\begin{array}{l}
d \eta_{1}=d \eta_{2}=0 \\
d \eta_{3}=\frac{1}{2}\left(\eta_{1} \wedge \eta_{2}+\eta_{1} \wedge \bar{\eta}_{2}\right) \\
d \eta_{4}=-\frac{\sqrt{-1}}{2}\left(\eta_{1} \wedge \eta_{2}-\eta_{1} \wedge \bar{\eta}_{2}\right) \\
d \eta_{5}=\frac{1}{2}\left(\eta_{1} \wedge \bar{\eta}_{3}+\bar{\eta}_{1} \wedge \eta_{3}\right)+\frac{\sqrt{-1}}{2}\left(\eta_{1} \wedge \bar{\eta}_{4}+\bar{\eta}_{1} \wedge \eta_{4}\right)
\end{array}\right.
$$

These equations are of type (2), which is equivalent to establishing (see Theorem 13) that the complex structure on $N^{10}$ is nilpotent.

\section{REFERENCES}

[A] M.F. Atiyah, Complex analytic connections in fibre bundles, Trans. Amer. Math. Soc. 85 (1957), 181-207. MR 19:172c

[AFGM] L.C. de Andrés, M. Fernández, A. Gray, J.J. Mencía, Compact manifolds with indefinite Kähler metrics, Proc. VIth Int. Coll. Differential Geometry (Ed. L.A. Cordero), Santiago (Spain) 1988, Cursos y Congresos Univ. Santiago de Compostela (Spain) 61 (1989), 2550. MR 91e:53066

[AGS] E. Abbena, S. Garbiero, S. Salamon, Hermitian geometry on the Iwasawa manifold, Boll. U.M.I. B (7) 11, no. 2, suppl. (1997), 231-249. [MR 98e:53121]

[BG] C. Benson, C. Gordon, Kähler and symplectic structures on nilmanifolds, Topology 27 (1988), 513-518. MR 90b:53042 
[Co] L.A. Cordero, Holomorphic principal torus bundles, curvature and compact complex nilmanifolds, Proc. Workshop on Curvature and Geometry (Ed. C.T.J. Dodson), Lancaster Univ. (U.K.) January 1989 (1989), 107-149. MR 92b:53111

[CFG1] L.A. Cordero, M. Fernández, A. Gray, Symplectic manifolds with no Kähler structure, Topology 25 (1986), 375-380. MR 87j:53051

[CFG2] L.A. Cordero, M. Fernández, A. Gray, Compact symplectic manifolds not admitting positive definite Kähler metrics, to appear, Proc. Int. Conf. on Topology and its Appl., Baku 3-9 October 1987, U.R.S.S..

[CFG3] L.A. Cordero, M. Fernández, A. Gray, The Frölicher spectral sequence and complex compact nilmanifolds, C. R. Acad. Sci. Paris Sér. I Math. 305 (1987), 753-756. MR 89c:32081

[CFG4] L.A. Cordero, M. Fernández, A. Gray, The Frölicher spectral sequence for compact nilmanifolds, Illinois J. Math. 35 (1991), 56-67. MR 92b:53112

[CFG5] L.A. Cordero, M. Fernández, A. Gray, The de Rham cohomology ring of a compact nilmanifold, preprint.

[CFGU] L.A. Cordero, M. Fernández, A. Gray, L. Ugarte, A general description of the terms in the Frölicher spectral sequence, Diff. Geom. and its Applic. 7 (1997), 75-84. MR 98m:53092

[DGMS] P. Deligne, P. Griffiths, J. Morgan, D. Sullivan, Real homotopy theory of Kähler manifolds, Invent. Math. 29 (1975), 245-274. MR 52:3584

[FG] M. Fernández, A. Gray, The Iwasawa manifold, (Differential Geometry, Peñíscola, 1985), Lect. Notes in Math. 1209 (1986), 157-159. MR 87k:53162

[F] A. Frölicher, Relations between the cohomology groups of Dolbeault and topological invariants, Proc. Nat. Acad. Sci. U.S.A. 41 (1955), 641-644. MR 17:409a

[GM] P. Griffiths, J. Morgan, Rational Homotopy Theory and Differential Forms, Progress in Math. 16, Birkhäuser, 1981. MR 82m:35014

[Ha] K. Hasegawa, Minimal models of nilmanifolds, Proc. Amer. Math. Soc. 106 (1989), 65-71. MR 89i:32015

[Hi] F. Hirzebruch, Topological Methods in Algebraic Geometry, third enlarged ed., SpringerVerlag, New York, 1966. MR 34:2573

[KN] S. Kobayashi, K. Nomizu, Foundations of Differential Geometry, I, II, Interscience Publ., New York, 1963, 1969. MR 27:2945 MR 38:6501

[Ko] K. Kodaira, On the structure of compact complex analytic surfaces, I, Amer. J. Math. 86 (1964), 751-798. MR 32:4708

[Ks] J.L. Koszul, Lectures on Fibre Bundles and Differential Geometry, Tata Inst., Bombay 1960. (Reissued Springer 1986.). MR 42:3698 MR 88e:53040

[Ma] I.A. Mal'cev, A class of homogeneous spaces, Amer. Math. Soc. Transl. 39 (1951). MR 10:50701, MR 12:589a

[McC] J. McCleary, User's Guide to Spectral Sequences, Publish or Perish, 1985. MR 87f:55014

$[\mathrm{Mu}] \quad$ S. Murakami, Sur certain espaces fibrés principaux holomorphes dont le groupe est abélien connexe, Osaka Math. J. 13 (1961), 143-167. MR 26:788

[Na] I. Nakamura, Complex parallelisable manifolds and their small deformations, J. Differential Geometry 10 (1975), 85-112. MR 52:14389

[NT] J. Neisendorfer, L. Taylor, Dolbeault Homotopy Theory, Trans. Amer. Math. Soc. 245 (1978), 183-210. MR 80f:32004

[No] K. Nomizu, On the cohomology of compact homogeneous spaces of nilpotent Lie groups, Ann. of Math. 59 (1954), 531-538. MR 16:218c

[R] M.S. Raghunathan, Discrete subgroups of Lie groups, Springer-Verlag, Berlin, 1972. MR 58:22394a

[Sa] Y. Sakane, On compact complex parallelisable solvmanifolds, Osaka J. Math. 13 (1976), 187-212. MR 54:10692

[Th] W.P. Thurston, Some simple examples of symplectic manifolds, Proc. Amer. Math. Soc. 55 (1976), 467-468. MR 53:6578

[Tr] A. Tralle, Applications of rational homotopy to geometry (results, problems, conjectures), Expo. Math. 14 (1996), 425-472. MR 97k:55015] 
[Va] V.S. Varadarajan, Lie Groups, Lie Algebras, and their Representations, Graduate Texts in Math. 102, Springer-Verlag, Berlin, 1984. MR 85e:22001

[Wa] H.C. Wang, Complex parallelisable manifolds, Proc. Amer. Math. Soc. 5 (1954), 771-776. MR 17:531a

Departamento de Geometría y Topología, Facultad de Matemáticas, Universidad de Santiago de Compostela, 15705 Santiago de Compostela, Spain

E-mail address: cordero@zmat.usc.es

Departamento de Matemáticas, Facultad de Ciencias, Universidad del País Vasco, Apartado 644,48080 Bilbao, Spain

E-mail address: mtpferol@lg.ehu.es

Department of Mathematics, University of Maryland, College Park, Maryland 20742

E-mail address: gray@bianchi.umd.edu

Departamento de Matemáticas (Geometría y Topología), Facultad de Ciencias, Universidad de Zaragoza, Campus Plaza San Francisco, 50009 Zaragoza, Spain

E-mail address: ugarte@posta.unizar.es 